VIII.

Aus der Internen Klinik der ungarischen Universität Kolozsvär

(Direktor: Prof. Dr. M. Jancsó).

\title{
Daten zur Wirkung der Extrakte einiger innerer Drüsen bei Osteomalazie.
}

(N-, Ca-, Mg- und P-Stoffwechseluntersuchungen.)

Von

Dr. Aladár Elfer, und Dr. J. Kappel,

klinisehem Adjunkt.

Universitätsassistent.

Auf Grund unserer vor Jahren gemachten Erfahrung, dass bei einem Falle von pathologischer Fettleibigkeit nach subkutaner Anwendung des infundibulären Extraktes der Hypophysis oine vorübergehende Zurückhaltung der $\mathrm{P}$-, $\mathrm{Ca}$ - und Mg-Elemente zu finden war, hielten wir schon damals, abgesehen von jeder Verallgemeinerung, die subkutane Anwendung dieses Stoffes bei denjenigen Krankheiten für geeignet, wo die Störung von Seiten des Knochengerüstes das Hauptcharakteristikum bildet. Wir dachten hauptsächlich an die Möglichkeit der ausgedehnteren Anwendung bei Osteomalazie und Rachitis. Da wir derartige experimentelle Erfahrungen bei unserem Krankenmateriale nicht sammeln konnten, hielten wir es für genügend, in erster Reihe die Frage beantworten zu können, ob wohl bei Osteomalazie auf subkutane Verabreichung des infundibulären T'eiles der Glandula pituitaria im Stoffwechsel der N-, Mg-, $\mathrm{Ca}-$ und P-Elemento besondere Abweichungen zu bemerken sind? Ob die eventuelle Retention der genannten Elemente, wie schon in einem Falle zu beobachten war, zu bemerken ist oder nicht?

Unsere diesbezüglichen Daten haben wir im vergangenen Jahre in der Festschrift zu Ehren der 25jährigen Tätigkeit des verstorbenen Herrn Universitätsprofessors Hofrat Dr. D. Szabó zusammenfassend aufgezeichnet. Doch wollen wir kurz auch bei dieser Gelegenhoit auf diese Daten zurückkommen, und zwar deshalb, weil wir die obige Frage gegenwärtig eigentlich darum in den Vordergrund stellen, um unscre Grundfrage, ob wir bei Osteomalazie nach Anwendung der Extrakte einzelner innerer Drüsen in der Exkretion obiger Elemente einen grösseren Unterschicd bemerken können, zu belcuchten. Und hier müssen wir auf Grund unserer bisherigen Erfahrungen eine derartige Wirkung des Extraktes vom hinteren Teile der Hypophysis kurz bekannt geben.

Abderbalden sagt in seinem "Lehrbuch der physiologischen Chemio" (II. Ausgabe, 1915, Seite 785): „Leider fehlen noch ausgedehnte Stoffwechselversuche bei der Osteomalazie und zwar vor und nach ihrem 
Ausbruch". Und ebenfalls Abderhal den erwähnt als einzig zuverlässige Daten diejenigen, wo die Ausscheidung des Ca die Einnahme bedeutend übertrifft. Als interessante Erscheinung erwähnt er im Gegensatze dazu, dass der Organismus das Mg-Element zurückbehält. Wenn auch nicht in diesem Sinne, so können wir doch in der deutschen und ungarischen Literatur über kurzdauernde genaue Stoffwechseluntersuchungen lesen bei ausgebildeter Osteomalazie. Diesbezügliche Daten hat Siegfried Neumann im Jahre 1894 und 1896 bekannt gemacht. Den Wert dieser Arbeit erhöhen seine mit Kastration verbundenen Versuche, wobei wir auch sehen können, in welcher Weise nach Kastration der negative Ca-Stoffwechsel die Retention aufweist (sein I. Fall). Auf Grund jener Bedingung, welche Abderhalden gerade bei Osteomalazie aufstellt, wäre es schwer, Stoffwechseluntersuchungen zu machen. Es ist bekannt, wie latent die Symptome bei Beginn der Krankheit sind und in jenem Stadium, wo die Osteomalazie klinisch vor uns steht, sind die mit Veränderung des Knochengerüstes einhergehenden und bisher für charakteristisch gehaltenen Störungen entweder schon abgelaufen oder im Ablaufe. Wir erwähnen eine Beobachtung ebenfalls von Neumann, wo bei einem schweren Falle von Osteomalazie in der Ausscheidung des Ca keine Störung zu finden war.

Die Aetiologie der Osteomalazie ist uns nicht bekannt. Der zu ihrer Pathogenese führende Weg aber ist im Aufbau. An die Namen von Pommer und Recklinghausen knüpfen sich bauptsächlich jene Untersuchungen, welche auf die anatomische Identität der Osteomalazie mit Rachitis hinweisen. Dem praktischen Arzte erscheint natürlich die Identifikation dieser zwei Krankheiten fremd, obzwar der grosse Kliniker Trousseau gerade auf Grund seiner klinischen Untersuchungen so entschieden sagt, dass die Erweichungsprozesse, welche sich im entwickelten oder in Entwicklung stehenden Knochengerüste zeigen, die gleichen sind, und dass die Unterschiede in den physiologischen Verhältnissen ihre Erklärung finden. Es wird genügen, wenn wir die so oft erwähnten Worte des grossen Virchow in Erinnerung bringen: "In der Osteomalazie wird wirklich resorbiert, Festes wird weich. In der Rachitis wird im wesentlichen nichts resorbiert, das Weiche wird nicht fest" (1853).

Die morphologischen Untersuchungen haben in die dualistische Auffassung Virchow's eine Lücke geschlagen. Nach den ausgedehnten Untersuchungen von Schmorl und den schon erwähnten von Pommer und Recklinkhausen kommt die Weichheit der Knochen in beiden Krankheiten nicht nur durch die pathologische Ca-Entziehung, Resorption (Halisterese) und den Zerfall der festen Knochensubstanz zu stande, sondern durch die Bildung der Knochengrundsubstanz, welche Ca nicht enthält (osteoid) und nicht über die Eigenschaft verfügt, die komplexe Kalziumverbindung $z u$ binden. Die übrigen eventuell zur Beobachtung kommenden Unterschiede, welche zwischen rachitischen und osteomalazischen Knochen zu sehen sind, wären mit einfachen Altersunterschieden zu erkJären. Danach ist es zu verstehen, dass unsere Kenntnisse der Pathogenese der Osteomalazie erst in der Entwicklung begriffen sind. Die jetzige Lage der morphologischen Richtung weist die Forscher auf jenen 
Weg, der zur Entwicklung der Krankheit führt. Klinisch sind die Erkrankungen an Osteomalazie viel seltener zu beobachten als die Rachitis. Im Hinblicke aber auf die morphologische Verwandtschaft können wir es nicht vermeiden, das in der Pathogenese der Rachitis Beobachtete auch bei Erforschung der Pathogenese der Osteomalazie zu Hilfe zu nehmen.

Im Jahre 1913, als wir die Wirkung des hinteren Teiles der Hypophysis im osteomalazischen Mineralstoffwechsel untersuchten, hielten wir uns nicht nur bloss das praktische Ziel vor Augen, dem von anderen schon in die Wege gesetzten praktischen Heilverfahren eine theoretische Unterlage zu geben, sondern wir wollten einigermassen Einblick gewinnen in den Verbrauch der wichtigeren Mineralstoffe, namentlich des $\mathrm{Ca}, \mathrm{Mg}$ und $\mathrm{P}$. Das Sammeln von Daten war eines unserer Hauptziele, welches bei osteomalazischer Erkrankung neben der Kenntnis der Pathogenese der Rachitis unbedingt notwendig ist. Unsere andere Frage, ob unter Einwirkung der verschiedenen inneren Drüsen und anderen chemischen Stoffe in der Ausscheidung der obigen Elemente eine auffallendere Abweichung zu finden ist, stützt sich auf die schon bei Rachitis bekannte Ansicht, dass die gleichmässige Funktion der inneren Drüsen im Aufbau der Knochensubstanz eine Notwendigkeit ist. Auch in der Pathogenese der Osteomalazie haben die inneren Drüsen eine wesentliche Rolle, dies beweisen die bisherigen Erfahrungen; daher sind jene Serienuntersuchungen, in welchen nach der Wirkung der Extrakte der inneren Drüsen auf die Ausscheidung der die Knochen aufbauenden Mineralstoffe bei Osteomalazie geforscht wird, wünschenswert. Insoweit wir uns aus der Literatur in dieser Frage orientieren konnten, wurden systematische Arbeiten nach jener Richtung nicht gemacht; wir fanden sogar, als wir im Jahre 1913 unsere Untersuchungen begannen, auch über Rachitis keine derartigen Daten. Die über Rachitis veröffentlichte gleichartige Arbeit von R. Bieling ist uns erst nach Beendigung unserer Stoffwechseluntersuchungen zur Kenntnis gekommen.

Unsere Mineralstoffwechselversuche machten wir nach den Regeln, welche wir in unseren Arbeiten gleicher Richtung schon wiederholt beschrieben haben. Wir waren ebenfalls neben der Berücksichtigung des Eiweissstoffwechsels auf die Ausscheidungsverhältnisse des $\mathrm{Ca}, \mathrm{Mg}$ und $\mathrm{P}$ bedacht. Der Inhalt der zugesetzten Stoffe an $\mathrm{N}, \mathrm{Ca}, \mathrm{Mg}$ und $\mathrm{P}$ war uns auf Grund eigener Untersuchungen bekannt. Den N-, Ca-, Mgund P-Gehalt des Urins und der Fäzes haben wir nach parallelen Bestimmungen in Rechnung gestellt. Die periodenweise gesammelten Urine haben wir in Glasgefässen aufbewahrt (Chloroform-Thymolaufbewahrung), bis wir ${ }^{*}$ die Mineralstoffe verarbeiten konnten, und die periodenweise gesammelten, getrockneten, abgewogenen und pulverisierten Fäzes wurden in mit Glasstopfen versehenen Glasgefässen bis zur Aufarbeitung aufbewahrt.

Die Stoffwechseluntersuchungen haben wir an einer 41 jährigen Frau (Bá.) gemacht. Es traten bei ihr schon vor dem Beginn des Versuches seit mehr als zwei Jahren heftige Kopfschmerzen auf. Im 5. Monat ihrer zehnten Gravidität kann sie schon kaum gehen. Ihr Zustand 
nach der Geburt ist wechselnd, jedoch schwerer. Vor ihrer Aufnahme liegt sie schon acht Monate im Bette. Die am Knochengerüste gefundenen Veränderungen verbunden mit dem klinischen Ablaufe berechtigen zur Annahme einer mittelschweren chronischen Osteomalazie.

Unsere Beobachtungen haben wir am 4.11. 1913 begonnen und die Kranke unter entsprechender Kontrolle bis zur Beendigung der Stoffwechseluntersuchungen, also bis 9.4.1914 gehalten. Ihr Körpergewicht beträgt im Anfange beiläufig 43 bis $44 \mathrm{~kg}$, und täglich bekommt sie etwa $1670 \mathrm{Kal}$. Wir hielten bei unserer Kranken rom 4. 11. bis 11. 11. Standarddiät ein und begannen erst dann mit unseren pünktlichen Stoffwechseluntersuchungen (Tab. I).

Ta belle I.

\begin{tabular}{|c|c|c|c|c|c|c|c|}
\hline \multirow{2}{*}{\multicolumn{2}{|c|}{$11 .-17.11 .1913$}} & \multirow{3}{*}{\begin{tabular}{|l} 
Einfuhr \\
32,38
\end{tabular}} & \multicolumn{3}{|c|}{ Ausscheidung } & \multicolumn{2}{|c|}{ Resultat } \\
\hline & & & \multirow{2}{*}{$\begin{array}{c}\begin{array}{c}\text { Urin } \\
?\end{array} \\
49,7\end{array}$} & \multirow{2}{*}{$\frac{\begin{array}{c}\text { läzes } \\
\text { ? }\end{array}}{2,56}$} & \multirow{2}{*}{$\frac{\begin{array}{c}\mathrm{Zu} \text { - } \\
\text { sammen }\end{array}}{52,26}$} & \multirow{2}{*}{$\begin{array}{c}\begin{array}{c}\mathrm{Zu}- \\
\text { sammen }\end{array} \\
+30,12\end{array}$} & \multirow{2}{*}{$\frac{\text { Täglich }}{+4,3}$} \\
\hline $\begin{array}{l}\text { Die vom Urin taiglieh } \\
\text { gemaclite Bestimmung }\end{array}$ & $N$ & & & & & & \\
\hline $\begin{array}{l}\text { Die rom } 7 \text { täg. ge- } \\
\text { mischten Urin gge- } \\
\text { machte Bestimmg. }\end{array}$ & $\begin{array}{l}\mathrm{Ca} \\
\mathrm{Mg} \\
\mathrm{P}\end{array}$ & $\begin{array}{r}11,17 \\
1,32 \\
11,84\end{array}$ & $\begin{array}{l}0,10 \\
0,35 \\
7,07\end{array}$ & $\begin{array}{l}3,34 \\
0,30 \\
1,88\end{array}$ & $\begin{array}{l}3,64 \\
0,65 \\
8,95\end{array}$ & $\begin{array}{r}+\quad, 03 \\
+\quad 0,67 \\
+\quad 2,89\end{array}$ & $\begin{array}{l}+1,07 \\
+0,09 \\
+0,4\end{array}$ \\
\hline
\end{tabular}

In diesem Falle finden wir unter den Stoffen, welche die Knochen aufbauen, in hohem Grade die Retention des Ca-Elementes; auch Retention des $\mathbf{P}$ ist zwar da, doch in kleinerem Masse als wir es beim $\mathrm{Ca}$ sehøn können, trotzdem der Wert der täglichen P-Zufuhr grösser ist als der des $\mathrm{Ca}(\mathrm{Ca}=1,59$ und $\mathrm{P}=1,69 \mathrm{~g})$ und zwar übertrifft der hauptsächlich mit dem Urin entfernte $\mathbf{P}$ auch das Zweifache des ausgeschiedenen Ca. Aus dieser einzigen Tatsache ist zu ersehen, dass ein Verhältnis in der Ausscheidung des $\mathrm{P}$ gegenüber dem Ca nicht immer besteht, und wir bemerken, dass bei der Untersuchung des Mineralstoffwechsels bei Rachitis die Frage der Ausscheidung des $\mathrm{P}$ und der Kohlensäure eine so wichtige Rolle zu spielen beginnt; ebenso muss es auch bei Osteomalazie sein. Mit Schloss bctonen auch wir, dass es wichtig ist, die Verhältnisse der Ausscheidung des $\mathrm{Ca}, \mathrm{P}$ und $\mathrm{CO}_{2}$ zusammen zu kennen, da dies jene Stoffe sind, welche die festen Substanzen der Knochen unzertrennlich aufbauen. Gassmann erwähnt in seinen Untersuchungen, welche sich auf die Zusammensetzung der gesunden und rachitischen Knochen beziehen: "Die in der Literatur verbreitete Tatsache, dass Kalkmangel die Ursache der Knochenrachitis sei, muss immer notwendigerweise dahin ergänzt werden, dass sich in gleicher Weise auch Phosphorsäure und Kohlensäure daran beteiligen; dadurch sind wir in der Lage, ein viel zutreffenderes, kompakteres Bild vom Wesen der Krankheit zu erhalten". Mit dem Herrn Chemiker Dr. Türk haben wir Untersuchungen gemacht, die Mineralstoffe gesunder und kranker Knochen betreffend; gegenwärtig können wir jedoch diese Daten nicht bringen, da unser Herr Kollege Türk am westlichen Kriegsschauplatze ist. Leider können wir in unserem jetzigen Falle, aber auch in den übrigen bei Osteomalaziekranken gemachten Untersuchungen 
nur für $\mathrm{Ca}$ und $\mathrm{P}$ mit Daten dienen. Die Aufarbeitung dieser Daten hat sich wegen des Ausbruches des Krieges vier Jahre verspätet. Und auch dieses haben wir nur mit fachkundiger Hilfe tun können. Bei diesen chemischen Analysen waren uns die Herren Chemiker Graf L. von Toldalagi und Herr Dr. Kössegi, Univ.-Assistent, eine grosse Hilfe. Ohne ihr Mitwirken hätten wir diese Daten heute nicht zusammenstellen können.

Zum Beweise, inwiefern die Ausscheidungswerte des Ca und P ohne jeden äusseren Einfluss im Verhältnis zu einander stehen, erwähnen wir unsere Fälle, in denen wir hinsichtlich der Exkretion der Mineralstoffe mit dem hinteren Teile der Hypophysis Versuche gemacht haben. Die Abhandlung hierüber folgt an anderer Stelle (Dr. EIfer).

Bei einer kranken Frau von 50 Jahren (Fr. Bi.), bei der ebenfalls eine chronische schwere Osteomalazie bestand, haben wir bei der im Juni 1914 vollzogenen Vorperiode, wie die Tab. II zeigt, folgende Daten erhalten:

T a b ell e II.

\begin{tabular}{|c|c|c|c|c|c|c|}
\hline \multirow[b]{2}{*}{$19 .-21.6 .1914$} & \multirow[b]{2}{*}{ Einfuhr } & \multicolumn{3}{|c|}{ Ausscheidung } & \multicolumn{2}{|c|}{ Resultat } \\
\hline & & $\begin{array}{c}\text { Urin } \\
2480 \mathrm{ccm}\end{array}$ & $\begin{array}{c}\text { Fäzes } \\
60 \mathrm{~g}\end{array}$ & $\begin{array}{c}\mathrm{Zu}- \\
\text { sammen }\end{array}$ & $\begin{array}{c}\mathrm{Zu-} \\
\text { sammen }\end{array}$ & Täglich \\
\hline $\begin{array}{c}\mathrm{N} \\
\mathrm{Ca}_{2} \\
\mathrm{Mg} \\
\mathrm{P}\end{array}$ & $\begin{array}{r}38,22 \\
4,14 \\
1,07 \\
4,98\end{array}$ & $\begin{array}{r}29,96 \\
0,24 \\
0,20 \\
2,40\end{array}$ & $\begin{array}{l}3,68 \\
1,41 \\
0,37 \\
0,91\end{array}$ & $\begin{array}{r}33,64 \\
1,65 \\
0,57 \\
3,31\end{array}$ & $\begin{array}{r}4,56 \\
2,49 \\
+0,50 \\
1,67\end{array}$ & $\begin{array}{l}1,52 \\
0,63 \\
0,16 \\
0,55\end{array}$ \\
\hline
\end{tabular}

Auch in diesem Falle ist die Retention bei allen drei Elementen za finden; doch ist die Zurückbehaltung des $\mathrm{Ca}$ noch immer günstiger, als die des $\mathrm{P}$, da gegenüber der Zufuhr von $1,38 \mathrm{~g}$ Ca die tägliche $\mathrm{Zu}-$ fuhr des P $1,66 \mathrm{~g}$ ist. Die Ursache der weniger günstigen Zurückbehaltung des $\mathrm{P}$ neben der żiemlich starken Retention des $\mathrm{N}$ sind wir geneigt, eher in der Erkrankung des Knochengerüstes zu suchen. Und wenn wir unseren III. Fall - Osteomalazie - (Frau Ve., 39 Jahre alt) in der ersten Periode betrachten, erhalten wir ohne jede Beeinflussung die unten stehenden Daten (Tab. III).

T a b e 11 e III.

\begin{tabular}{|c|c|c|c|c|c|c|}
\hline \multirow[b]{2}{*}{$10 .-12.7 .1919$} & \multirow{2}{*}{ Einfuhr } & \multicolumn{3}{|c|}{ Ausscheidung } & \multicolumn{2}{|c|}{ Resultat } \\
\hline & & $\begin{array}{l}\text { Urin } \\
2600 \mathrm{ccm}\end{array}$ & $\begin{array}{l}\text { Fäzes } \\
70 \mathrm{~g}\end{array}$ & $\begin{array}{c}\mathrm{Zu-} \\
\text { sammen }\end{array}$ & $\begin{array}{c}\mathrm{Zu}- \\
\text { sammen }\end{array}$ & Täglich \\
\hline $\begin{array}{c}\mathrm{N} \\
\mathrm{Ca} \\
\mathrm{Mg}\end{array}$ & $\begin{array}{r}40,08 \\
3,95 \\
1,13\end{array}$ & $\begin{array}{r}28,36 \\
0,30 \\
0,16\end{array}$ & $\begin{array}{l}3,13 \\
3,14 \\
0,63\end{array}$ & $\begin{array}{r}31,49 \\
3,44 \\
0,79\end{array}$ & $\begin{array}{l}8,59 \\
0,49 \\
0,34\end{array}$ & $\begin{array}{l}2,63 \\
0,13 \\
0,11\end{array}$ \\
\hline
\end{tabular}

In diesem Falle können wir unsere Werte anders beurteilen. Neben der starken Zurückbehaltung des $\mathrm{N}$ ist die des $\mathrm{Ca}$ eine geringere und die Retention des $\mathrm{P}$ ist demgemäss stärker. Doch ist neben dem $1,31 \mathrm{~g}$ $\mathrm{Ca}$ der täglichen Einfuhr an P $1,84 \mathrm{~g}$ zurückbehalten worden, es kann die um $0,50 \mathrm{~g}$ grössere Zufuhr des $\mathrm{P}$ die um ein wenig grössere P-Retention einigermassen erklären. Beweise hierfür aber fehlen. 
Unser IV. Fall bezieht sich auf einen wahrscheinlich an Chondrodystrophie erkrankten kleinen Knaben, bei dem wir ebenfalls den Extrakt des hinteren Teiles der Hypophysis zur Behandlung angewendet haben. Die 7 tägige Vorperiode ist aus der Tabelle IV zu ersehen.

T a belle IV. (Mi.)

\begin{tabular}{|c|c|c|c|c|c|c|}
\hline \multirow{2}{*}{ 10.-17. 3. 1913} & \multirow{2}{*}{ Einfuhr } & \multicolumn{3}{|c|}{ A usscheidung } & \multicolumn{2}{|c|}{ Resultat } \\
\hline & & $\begin{array}{l}\text { Urin } \\
3010 \mathrm{ccm}\end{array}$ & $\begin{array}{l}\text { Fäzes } \\
163 \mathrm{~g}\end{array}$ & $\begin{array}{c}\mathrm{Zu-} \\
\text { sammen }\end{array}$ & $\begin{array}{c}\mathrm{Zu-} \\
\text { sammen }\end{array}$ & Täglich \\
\hline $\begin{array}{c}\mathrm{N} \\
\mathrm{Ca} \\
\mathrm{Mg} \\
\mathrm{P}\end{array}$ & $\begin{array}{c}89,93 \\
6,989 \\
1,60 \\
10,42\end{array}$ & $\begin{array}{l}60,35 \\
0,227 \\
0,046(?) \\
4,003\end{array}$ & $\begin{array}{c}16,11 \\
2,054 \\
0,256 \\
2,787\end{array}$ & $\begin{array}{l}76,46 \\
2,281 \\
0,302 \\
6,79\end{array}$ & $\begin{array}{c}13,49 \\
4,708 \\
1,298 \\
3,63\end{array}$ & $\begin{array}{l}1,92 \\
0,67 \\
0,186 \\
0,51\end{array}$ \\
\hline
\end{tabular}

Eine grössere Retention des $\mathrm{Ca}$ als die des $\mathrm{P}$ sehen wir auch in diesem Falle (die tägliche Ca-Einfuhr ist $0,99 \mathrm{~g}$, währenddem die tägliche P-Einfuhr $1,48 \mathrm{~g}$ ist), auch wenn wir nur die Resultate der Ausscheidungswerte in Betracht nehmen. Wenn wir aber die bei der Einfuhr gefundenen Zahlen untersuchen, ist wiederum die tägliche P-Zufuhr um $0,5 \mathrm{~g}$ grösser. Weiteres folgern wir aus diesen Daten auch schon auf Grund der vorhergehenden natürlich nicht.

Endlich erwähnen wir unsere Daten, dic wir zwecks der Vergleichung bei einer 24jährigen kranken Frau - mit gesundem Knochengerüst - gewonnen haben.

Tabelle V. (Flora Flo.)

\begin{tabular}{c|c|r|r|r|r|r}
\hline \multirow{3}{*}{ 2.-4. 7. 1914 } & \multirow{2}{*}{ Einfuhr } & \multicolumn{2}{|c|}{ A us seheidung } & \multicolumn{2}{|c}{ Resultat } \\
\cline { 3 - 7 } & & $\begin{array}{c}\text { Urin } \\
2800 \mathrm{ccm}\end{array}$ & $\begin{array}{c}\text { Fäzes } \\
79 \mathrm{~g}\end{array}$ & $\begin{array}{c}\text { Zu- } \\
\text { sammen }\end{array}$ & $\begin{array}{r}\text { Zu- } \\
\text { sammen }\end{array}$ & Täglich \\
\hline $\mathrm{N}$ & 50,55 & 41,35 & 3,43 & 44,78 & 5,77 & 1,92 \\
$\mathrm{Ca}$ & 6,0 & 0,64 & 5,39 & 6,03 & $-0,03$ & $-0,01$ \\
$\mathrm{Mg}$ & 1,47 & 0,23 & 0,67 & 0,90 & 0,57 & 0,19 \\
$\mathrm{P}$ & 7,24 & 2,85 & 2,60 & 5,45 & 1,79 & $+0,59$
\end{tabular}

Der täglichen Ca-Einfuhr von $2,0 \mathrm{~g}$ gegenüber steht die Einfuhr von $2,41 \mathrm{~g} \mathrm{P}$. Die Bilanz des $\mathrm{Ca}$ ist also viel ungünstiger. Jedenfalls können wir auch aus diesen fünf Tabellen ersehen, dass wir bei dem Gegenüberstellen der Werte und bei der Folgerung von Daten der Ausscheidung des einen Elementes auf die des anderen die grösste Vorsicht beobachten müssen, obzwar wir in diesen Fällen bei unscren Kranken (ausgenommen den IV. Eall) vor Beginn der Stoffwechseluntersuchungen kürzere oder längere Zeit schon Standarddiät einhielten.

Unsere ersten experimentellen Serien haben wir mit dem Extrakt des hinteren Teiles der Hypophysis gemacht. Mit diesem Stoffe gewonnene Daten stehen uns in ziemlich grosser Zahl zur Verfügung, so dass wir sagen können, dass - aus Mangel von verschiedenen anderen an Osteomalaziekranken gemachten Kontrollversuchen - auch diese Serie, ebenso wie ein grosser Teil unserer unten zu erwähnenden Serien sich in negativer Richtung entschieden hat. 
In unserem ersten Falle gaben wir nach der 7 tägigen Vorperiode während 21 Tagen täglich $1 \mathrm{ccm}$ von Richter's Glanduitrin (entspricht $0,2 \mathrm{~g}$ frischen Stoffes) subkutan. Die 21 tägige Serie teilten wir in drei Perioden (7tägige) ein, aber die Tabelle (Tab. VIa) geben wir zusammen.

Ta a ell e VIa. (Fr. Bá.)

\begin{tabular}{|c|c|c|c|c|c|c|c|}
\hline \multirow{2}{*}{\multicolumn{2}{|c|}{ 18. 11.-8. 12.1913}} & \multirow{3}{*}{$\begin{array}{l}\text { Einfuhr } \\
246,95\end{array}$} & \multicolumn{3}{|c|}{ Ausscheidung } & \multicolumn{2}{|c|}{ Resultat } \\
\hline & & & \multirow{2}{*}{\begin{tabular}{|c|}
$\begin{array}{c}\text { Urin } \\
9070 \mathrm{~cm}\end{array}$ \\
146,27
\end{tabular}} & \multirow{2}{*}{$\begin{array}{r}\text { Fäzes } \\
78 \mathrm{~g}\end{array}$} & \multirow{2}{*}{$\frac{\begin{array}{c}\mathrm{Zu}- \\
\text { sammen }\end{array}}{149,01}$} & \multirow{2}{*}{$\frac{\begin{array}{c}\mathrm{Zu-} \\
\text { sammen }\end{array}}{97,94}$} & \multirow{2}{*}{$\frac{\text { Täglich }}{4,66}$} \\
\hline $\begin{array}{l}\text { Im Urin tägliche } \\
\text { N-Bostimmung }\end{array}$ & $\mathrm{N}$ & & & & & & \\
\hline $\begin{array}{l}\text { Die vom } 7 \text { tüg. ge- } \\
\text { mischten Urin ge- } \\
\text { machte Bestimmg. }\end{array}$ & $\begin{array}{c}\mathrm{Ca} \\
\mathrm{Mg} \\
\mathrm{P}\end{array}$ & $\begin{array}{r}32,35 \\
3,90 \\
35,27\end{array}$ & $\begin{array}{l}0,65 \\
0,65 \\
9,64\end{array}$ & $\begin{array}{l}7,19 \\
0,59 \\
3,97\end{array}$ & $\begin{array}{r}7,84 \\
1,24 \\
13,61\end{array}$ & $\begin{array}{r}24,51 \\
2,66 \\
21,66\end{array}$ & $\begin{array}{l}1,16 \\
0,12 \\
1,03\end{array}$ \\
\hline
\end{tabular}

Wenn wir die täglich zurückbehaltenen Werte der Vorperiode gegenüberstellen, sehen wir aus dieser 21 tägigen Serie eine Retention sämtlicher untersuchten Elemente in grösserem oder kleinerem Masse. Die tägliche Retention ist im Durchschnitte beim $\mathrm{N}$ um $30 \mathrm{cg}$, beim $\mathrm{Ca} \mathrm{um}$ $9 \mathrm{cg}$, beim $\mathrm{Mg}$ durehsehnittlich um $3 \mathrm{cg}$ und beim $\mathrm{P}$ um $63 \mathrm{cg}$ höher als in der Grundperiode. Da von einer 21 tägigen Untersuchung die Rede ist, sind diese Daten einigermassen zu verwerten, und wir betonen besonders die Zurückhaltung zwischen $\mathrm{P}$ and $\mathrm{Ca}$, denn die Unregelmässigkeit, welche sich in der Grundperiode bei der Retention des P zeigt, gleicht sich in der Glanduitrin-Periode einigermassen aus, indem jetzt das täglich zurückbehaltene Quantum des $\mathrm{P}$ viel grösser wird; dies ist das Ergebnis dessen, dass mit dem Urin weniger $\mathrm{P}$ ausgeschieden wird.

In der III. Nachperiode lassen wir die Kranke zehn Tage lang unbeeinflusst, so dass wir auf eine andere Beobachtungsserie übergehen können.

T a belle IVb. (Fr. Bá.)

\begin{tabular}{|c|c|c|c|c|c|c|c|}
\hline \multirow{2}{*}{\multicolumn{2}{|c|}{ 9.-19. 12. 1913}} & \multirow{2}{*}{ Einfuhr } & \multicolumn{3}{|c|}{ Aussebeidung } & \multicolumn{2}{|c|}{ Resultat } \\
\hline & & & $\begin{array}{c}\text { Urin } \\
6675 \mathrm{ccm}\end{array}$ & $\begin{array}{l}\text { Fäzes } \\
100 \mathrm{~g}\end{array}$ & $\begin{array}{c}7 u- \\
\text { sammen }\end{array}$ & $\begin{array}{c}\text { Zu- } \\
\text { sammen }\end{array}$ & Täglieh \\
\hline \multirow{2}{*}{$\begin{array}{c}\text { Tiggliche Bestimmung } \\
\text { des N im Urin } \\
\text { Die Mineralstoffe } \\
\text { wurden vom ge- } \\
\text { mischten Urin be- } \\
\text { stimmt }\end{array}$} & $\mathrm{N}$ & 119,42 & 77,27 & 3,92 & 81,19 & 37,23 & 3,72 \\
\hline & $\begin{array}{c}\mathrm{Ca} \\
\mathrm{Mg} \\
\mathrm{P}\end{array}$ & $\begin{array}{r}15,88 \\
1,92 \\
17,24\end{array}$ & $\begin{array}{l}0,40 \\
0,12 \\
5,90\end{array}$ & $\begin{array}{l}9,12 \\
0,92 \\
4,29\end{array}$ & $\begin{array}{r}9,52 \\
1,04 \\
10,19\end{array}$ & $\begin{array}{l}6,36 \\
0,88 \\
7,05\end{array}$ & $\begin{array}{l}0,63 \\
0,08 \\
0,7\end{array}$ \\
\hline
\end{tabular}

In der 10 tägigen Pause finden wir bei den untersuchten Elementen durchschnittlich Retention, doch ist deren Wert - ausgenommen für das $\mathrm{P}$ - noch kleiner wie in der Grundperiode; beim $\mathrm{P}$ ist die günstigere Retention auch jetzt noch zu sehen. Aus diesem einzigen Befunde können wir jedoch über die Wirkung des hinteren T'eiles der Hypophysis auf obige Stoffe bei Osteomalazie nichts Bestimmtes aussagen. Das Gegenüberstellen der Vor- und Nachperiode erlaubt uns zwar eine Vergleichung, doch wollen wir nicht vergessen, dass wir zur Unter- 
Daten zur Wirkung der Extrakte einiger innerer Drüsen bei Osteomalazie. 111

suchung eine Osteomalaziekranke wählten, bei der uns gerade die Ausscheidungsverhältnisse der genannten Stoffe noch zu wenig klar sind, um die kurze Zeit dauernden Versuche in günstiger oder ungünstiger Richtung mit Bestimmtheit auf eine Einwirkung des Versuches zurückführen zu können. Bei Verwendung von mehreren Fällen lassen sich natürlich strengere Folgerungen ziehen.

Bei unserer II. Osteomalaziekranken (Frau Bi.) haben wir täglich während 19 Tage $1 \mathrm{ccm}$ englisches Präparat (Parke, Davis u. Co.) subkutan verabreicht. Unsere Bestimmungen haben wir am ersten und an den letzten drei Tagen der Pituitrinperiode gemacht, wie dies folgende Tabellen zeigen.

Tabelle VIL a. (Fr. Bi.)

\begin{tabular}{|c|c|c|c|c|c|c|}
\hline \multirow{2}{*}{ 6.-8. 7. 1914} & \multirow{2}{*}{ Einfuhr } & \multicolumn{3}{|c|}{ Ausscheidung } & \multicolumn{2}{|c|}{ Resultat } \\
\hline & & $\begin{array}{c}\text { Urin } \\
2440 \mathrm{ccm}\end{array}$ & $\begin{array}{l}\text { Fäzes } \\
77 \mathrm{~g}\end{array}$ & $\begin{array}{c}\mathrm{Zu}- \\
\text { sammen }\end{array}$ & $\begin{array}{c}\mathrm{Zu}- \\
\text { sammen }\end{array}$ & Täglich \\
\hline $\mathrm{N}$ & 38,20 & 26,59 & 5,23 & 31,82 & 6,38 & 2,12 \\
\hline $\mathrm{Ca}$ & 4,15 & 0,22 & 1,43 & 1,65 & 2,50 & 0,83 \\
\hline $\mathrm{Mg}$ & 1,06 & 0,26 & 0,17 & 0,43 & 0,64 & 0,21 \\
\hline$P^{\circ}$ & 4,96 & 2,29 & 0,85 & 3,14 & 1,82 & 0,60 \\
\hline
\end{tabular}

T a belle VIIb. (Fr. Bi.)

\begin{tabular}{|c|c|c|c|c|c|c|}
\hline \multirow{2}{*}{$22 \cdot-24.7 .1814$} & \multirow{2}{*}{ Einfuhr } & \multicolumn{3}{|c|}{ Ausscheidung } & \multicolumn{2}{|c|}{ Resultat } \\
\hline & & $\begin{array}{c}\text { Urin } \\
2750 \mathrm{ccm}\end{array}$ & $\begin{array}{l}\text { Fäzes } \\
89 \mathrm{~g}\end{array}$ & $\begin{array}{c}\mathrm{Zu}- \\
\text { sammen }\end{array}$ & $\begin{array}{c}\mathrm{Zu}- \\
\text { sammen }\end{array}$ & Täglich \\
\hline $\mathrm{N}$ & 38,22 & 28,71 & 5,82 & 34,53 & 3,69 & 1,23 \\
\hline $\mathrm{Ca}$ & 4,14 & 0,22 & 1,60 & 1,82 & 2,32 & 0,77 \\
\hline $\mathrm{Mg}$ & 1,06 & 0,26 & 0,21 & 0,47 & 0,59 & $+0,19$ \\
\hline$P$ & 4,98 & 2,29 & 1,17 & 3,46 & 1,52 & 0,50 \\
\hline
\end{tabular}

Wenn wir unsere Untersuchungen in diesem Falle kurz zusammenfassen, können wir in der Pituitrin-Periode nur eine sehr geringe Retention des $\mathrm{Ca}, \mathrm{Mg}$ und $\mathrm{P}$ sehen. Diese Daten erwähnen wir zur Vergleichung mit dem ersten Falle. Die letzte Periode konnten wir leider wegen Ausbruchs des Krieges nur 3 Tage lang beobachten.

Tabelle VIIc. (Fr. Bi.)

\begin{tabular}{|c|c|c|c|c|c|c|}
\hline \multirow{2}{*}{$25 .-27.7 .1914$} & \multirow{2}{*}{ Einfuhr } & \multicolumn{3}{|c|}{ Ausscheidung } & \multicolumn{2}{|c|}{ Resultat } \\
\hline & & $\begin{array}{c}\text { Urin } \\
2640 \mathrm{ccm}\end{array}$ & $\begin{array}{l}\text { Fäzes } \\
21 \mathrm{~g}\end{array}$ & $\begin{array}{c}\mathrm{Zu-} \\
\text { sammen }\end{array}$ & $\begin{array}{c}\mathrm{Zu}- \\
\text { sammen }\end{array}$ & Täglich \\
\hline $\begin{array}{c}\mathrm{N} \\
\mathrm{Ca} \\
\mathrm{Mg} \\
\mathrm{P}\end{array}$ & $\begin{array}{r}38,22 \\
4,14 \\
1,06 \\
4,98\end{array}$ & $\begin{array}{r}30,78 \\
0,24 \\
0,10 \\
2,54\end{array}$ & $\begin{array}{l}1,40 \\
0,42 \\
0,11 \\
0,28\end{array}$ & $\begin{array}{r}32,18 \\
0,66 \\
0,22 \\
2,82\end{array}$ & $\begin{array}{l}6,04 \\
3,48 \\
0,84 \\
2,16\end{array}$ & $\begin{array}{l}2,01 \\
1,12 \\
0,26 \\
0,72\end{array}$ \\
\hline
\end{tabular}

Wir weisen nur auf die kurze Aufzählung dieser Nachperiode hin.

Bei der III. osteomalaziekranken Frau gaben wir während der Versuchszeit täglich $1 \mathrm{ccm}$ englisches Präparat (Parke, Davis u. Co.) von der Voraussetzung ausgehend, dass, wenn wir auf diese Weise mit 
einem grösseren Quantum des wirkenden Stoffes arbeiten, auch die eventuelle Wirkung eher zu erkennen sei.

Ta belle VIII a. (F. Ve.)

\begin{tabular}{|c|c|c|c|c|c|c|}
\hline \multirow{2}{*}{$20 .-22.7 .1914$} & \multirow{2}{*}{ Einfuhr } & \multicolumn{3}{|c|}{ Ausseheidung } & \multicolumn{2}{|c|}{ Resultat } \\
\hline & & $\begin{array}{c}\text { Urin } \\
2165 \mathrm{cem}\end{array}$ & $\begin{array}{l}\text { Fäzes } \\
60 \mathrm{~g}(?)\end{array}$ & $\begin{array}{c}\mathrm{Zu-} \\
\text { sammen }\end{array}$ & $\begin{array}{c}\text { Zu- } \\
\text { sammen }\end{array}$ & Täglich \\
\hline $\mathrm{N}$ & 38,34 & 28,15 & 1,92 & 30,07 & 8,27 & 2,76 \\
\hline $\mathrm{Ca}$ & 3,91 & 0,28 & 3,54 & 3,82 & 0,09 & 0,03 \\
\hline $\mathrm{Mg}$ & 1,13 & 0,20 & 0,13 & 0,33 & 0,80 & 0,26 \\
\hline $\mathrm{P}$ & 5,38 & 2,38 & 1,41 & 3,79 & 1,59 & 0,53 \\
\hline
\end{tabular}

In dieser kurzen Periode begegnen wir zuerst bei subkutaner Anwendung des Pituitrins einer kleineren $\mathrm{Ca}$-Retention als in der Grundperiode; aber auch das ist $\mathrm{zu}$ betonen, dass bei dieser unserer ersten Untersuchung das Quantum des wirkenden Stoffes auf das Zweifache erhöht wurde. Wir müssen aber im Zusammenhange mit dem Sinken der Ca-Retention erwähnen, dass der Retentionswert des $\mathrm{Mg}$ der zweifache des in der Grundperiode gefundenen Wertes ist. Jedenfalls betonen wir die Fehlerhaftigkeit auch dieser Experimentserie, da nach unseren Notizen das Sammeln der Fäzes nicht mit absoluter Genauigkeit geschehen ist. Dies beeinflusst aber die Richtung der Ausscheidungzahlen nicht. Und für weitere Folgerungen könnten wir die Daten einer Versuchsserie ohnehin nicht verwenden.

Die Nachperiode hat in diesem Falle wieder drei Tage lang gedauert.

Tabelle VIII b.

\begin{tabular}{|c|c|c|c|c|c|c|}
\hline \multirow{2}{*}{ 23.-25. 7. 1914} & \multirow{2}{*}{ Einfuhr } & \multicolumn{3}{|c|}{ A ussehejdung } & \multicolumn{2}{|c|}{ Resultat } \\
\hline & & $\begin{array}{c}\text { Urin } \\
?\end{array}$ & $\begin{array}{c}\text { Fäzes } \\
50 \mathrm{~g}\end{array}$ & $\begin{array}{c}\mathrm{Zu}- \\
\text { sammen }\end{array}$ & $\begin{array}{c}1 / 1 \mathrm{u}- \\
\text { sammen }\end{array}$ & Täglich \\
\hline $\mathrm{N}$ & 37,47 & 31,53 & 2,09 & 33,62 & 3,85 & 1,26 \\
\hline $\mathrm{Ca}$ & 3,90 & 0,30 & 2,71 & 3,01 & 0,89 & 0,29 \\
\hline $\mathrm{Mg}$ & 1,13 & 0,24 & 0,43 & 0,67 & 0,49 & 0,15 \\
\hline P & 5.31 & 2,49 & 1,23 & 3,72 & 1,59 & 0,53 \\
\hline
\end{tabular}

In dieser Serie sehen wir betreffs der Ca-Retention eine viel günstigere Zahl, obzwar wir hier sagen müssen, dass wir die Daten des Urins vom 23. freiwillig in die Serie stellten, auf Grund des am 24. und 25. extra bestimmten Urinbefundes. Diese freiwillige Annahme beeinflusst den Endwert unserer Daten, die Ca-Ausschiedung betreffend, nicht.

Unser IV. am Knochengerüste erkrankter Patient bekommt während einer 45 tägigen Versuchsperiode täglich $1 \mathrm{ccm}$ Richter's Glanduitrin subkutan. Während der Behandlung hat sich der Zustand des Kranken nicht verschlechtert. Betreffs der Wirkungsweise möchten wir es nicht wagen uns im Zusammenhange mit diesem Falle zu äussern. Die Stoffwechseltabelle geben wir in dieser Arbeit zusammengefasst, doch erwähnen wir, dass wir unsere Daten ursprünglich in 6 einzeln stehenden Perioden noch im Jahre 1913 aufgearbeitet haben. 
Daten zur Wirkung der Extrakte einiger innerer Drüsen bei Osteomalazie. 113

Tabelle IXa.

\begin{tabular}{|c|c|c|c|c|c|c|c|}
\hline \multirow{2}{*}{\multicolumn{2}{|c|}{ 17. 3.-30. 4. 1913}} & \multirow{2}{*}{ Einfuhr } & \multicolumn{3}{|c|}{ A usseheidung } & \multicolumn{2}{|c|}{ Resultat } \\
\hline & & & $\begin{array}{c}\text { Urin } \\
24280 \mathrm{ccm}\end{array}$ & $\begin{array}{l}\text { Fäzes } \\
740 \mathrm{~g}\end{array}$ & $\begin{array}{c}\text { \%u- } \\
\text { sammen }\end{array}$ & $\begin{array}{c}\text { Zu- } \\
\text { saminen }\end{array}$ & Taglich \\
\hline $\begin{array}{l}\text { Tugliche } \mathrm{N} \text { - Bestimmg } \\
\text { vom Drin }\end{array}$ & $\mathrm{N}$ & 553,82 & 499,14 & 35,08 & 534,22 & 19,60 & 0,43 \\
\hline $\begin{array}{l}\text { Die Bestimmg. der } \\
\text { Mineralstoffe wurde }\end{array}$ & $\mathrm{Ca}$ & 44,49 & 1,09 & 11,37 & 12,46 & 32,03 & 0.71 \\
\hline aus den periudert- \{ & $\mathrm{Mg}$ & 10,24 & 1,78 & 1,69 & 3,47 & 6,77 & 0,15 \\
\hline $\begin{array}{l}\text { weis resammelten } \\
\text { Urin gemaclit. }\end{array}$ & $\mathrm{N}$ & 63,03 & 29,88 & 18,89 & 48,77 & 14,26 & 0,31 \\
\hline
\end{tabular}

Im Verhältnis zur Grundperiode sind die Werte der tägliehen Retention bei sämtlichen Elementen mit Ausnahme des $\mathrm{Ca}$ niedriger, doch ist ebendann die N-Retention viel geringer. Obzwar die Versuchsperiode 45 Tage lang dauerte, sind unsere Daten zu Schlüssen in einer bestimmten Richtung nicht geeignet. Die Nachperiode hat 6 Tage gedauert.

$\mathrm{Tab}$ e lle $\mathrm{IX} b$.

\begin{tabular}{|c|c|c|c|c|c|c|c|}
\hline \multirow{2}{*}{\multicolumn{2}{|c|}{ 5. 1.-5. 6. 1913}} & \multirow[b]{2}{*}{ Einfuhr } & \multicolumn{3}{|c|}{ Ausscheidungr } & \multicolumn{2}{|c|}{ Resultat } \\
\hline & & & $\begin{array}{c}\text { Urin } \\
3425 \mathrm{ccm}\end{array}$ & $\begin{array}{l}\text { Fäzes } \\
117 \mathrm{~g}\end{array}$ & $\begin{array}{c}\text { Zu- } \\
\text { sammen }\end{array}$ & $\begin{array}{c}Z u^{-} \\
\text {sammed }\end{array}$ & Täglich \\
\hline $\begin{array}{l}\text { Targliche N-Bestimmg } \\
\text { in Urin }\end{array}$ & $N$ & 81,06 & 69,19 & 7,63 & 76,52 & 4,54 & 0,75 \\
\hline $\begin{array}{c}\text { Die Bestimraug der } \\
\text { Mineralstoffe ist aus } \\
\text { dem gemischten Urin } \\
\text { gemacht worden }\end{array}$ & $\begin{array}{r}\mathrm{Ca} \\
\mathrm{Mg} \\
\mathrm{P}\end{array}$ & $\begin{array}{l}6,02 \\
1,38 \\
8,64\end{array}$ & $\begin{array}{l}0,159 \\
0,33 \\
3,90\end{array}$ & $\begin{array}{l}1,82 \\
0,26 \\
2,98\end{array}$ & $\begin{array}{l}1,979 \\
0,59 \\
6,88\end{array}$ & $\begin{array}{l}4,04 \\
0,79 \\
1,76\end{array}$ & $\begin{array}{l}0,67 \\
0,13 \\
0,26\end{array}$ \\
\hline
\end{tabular}

Die Retentionswerte sind im Verhältnis zur Grundperiode zwar kleiner, doch sind auch diese-Werte zu Schlüssen in irgendeiner Richtung nicht geeignet.

Endlich erwähnen wir unsere Daten, welche wir bei einer kranken Frau mit gesundem Knochengerüste auf die Weise gewonnen haben, dass wir während 3 Tagen der Kranken täglich $2 \mathrm{cem}$ von dem aus dem vorderen Teile der Hypophysis gewonnenen Extrakte subkutan verabreichten.

Ta b e lle Xa.

\begin{tabular}{|c|c|c|c|c|c|c|}
\hline \multirow[b]{2}{*}{ 14.-16. 7. 1914} & \multirow{2}{*}{ Einfuhr } & \multicolumn{3}{|c|}{ Ausscheidung } & \multicolumn{2}{|c|}{ Resultat } \\
\hline & & $\begin{array}{c}\text { Urin } \\
2500 \mathrm{ccm}\end{array}$ & $\begin{array}{c}\text { Fäzes } \\
82 \mathrm{~g}\end{array}$ & $\begin{array}{c}7 \mathrm{u}- \\
\text { sammen }\end{array}$ & $\begin{array}{c}\mathrm{Zu}- \\
\text { sammen }\end{array}$ & Täglich \\
\hline $\begin{array}{c}\mathrm{N} \\
\mathrm{Ca} \\
\mathrm{Mg} \\
\mathrm{P}\end{array}$ & $\begin{array}{r}47,49 \\
5,61 \\
1,39 \\
6,80\end{array}$ & $\begin{array}{r}36,76 \\
0,64 \\
0,28 \\
2,40\end{array}$ & $\begin{array}{l}4,27 \\
6,34 \\
0,69 \\
3,06\end{array}$ & $\begin{array}{r}41,03 \\
6,98 \\
0,97 \\
5,46\end{array}$ & $\begin{array}{r}6,46 \\
-1,37 \\
0,42 \\
1,34\end{array}$ & $\begin{array}{r}+2,15 \\
-0,68 \\
+0,14 \\
+0,44\end{array}$ \\
\hline
\end{tabular}

In dieser kurzen Serie fällt der Verlust an Ca auf. Es handelt sich täglich um einen grösseren Wert als ein halbes Gramm. Wir wagen jedoch nicht bestimmte Folgerungen daraus zu ziehen, da wir wieder einen anderen Fall untersuchten, bei dem in einer dreitägigen Serie in der Vorperiode nicht einmal Spuren der Ca-Retention zu sehen waren. 
Die Nachperiode können wir zur Vergloichung heranziehen, doch müssen wir bemerken, dass wir den glandulären Teil der Hypophysis, täglich $1 \mathrm{eem}$, der Kranken weiter bis zum 25. 7: verabreichten. Da jedoch die Daten der letzten 3 Tage mangelhaft waren und zur gleichen Zeit auch Menstruation auftrat, haben wir die so gewonnenen Werte nicht benutzt.

Tabeltexb.

\begin{tabular}{|c|c|c|c|c|c|c|}
\hline \multirow[b]{2}{*}{$26 .-28.7 .1914$} & \multirow[b]{2}{*}{ Finfuhr } & \multicolumn{3}{|c|}{ Ausscheidung } & \multicolumn{2}{|c|}{ Resultat } \\
\hline & & $\begin{array}{c}\text { Urin } \\
2040 \mathrm{ccm}\end{array}$ & $\begin{array}{c}\text { Fäzes } \\
78 \mathrm{~g}\end{array}$ & $\begin{array}{l}\text { /u- } \\
\text { sammen }\end{array}$ & $\begin{array}{c}\text { Zu- } \\
\text { sammen }\end{array}$ & 'Tüglich \\
\hline $\mathrm{N}$ & 38,93 & 28,96 & 4,17 & 33,13 & 5.80 & 1,93 \\
\hline $\mathrm{Ca}$ & 5,96 & 0,47 & 5,29 & 5,76 & 0,20 & $+0,06$ \\
\hline $\mathrm{Mg}$ & 1,47 & 0,24 & 0,58 & 0,82 & 0,65 & 0,21 \\
\hline $\mathrm{P}$ & 7,08 & 2,13 & 2,94 & 5,07 & 2,01 & 0,67 \\
\hline
\end{tabular}

Der Ca-Verlust, wie er bei Versuchstieren zu finden ist, ist verschwunden und wir können im Zusammenhange mit der Retention der übrigen Stoffe (N und $\mathrm{P}$ ) fast Gleichgewicht in der Ausscheidung des Ca erkenuen.

Bieling machte auf gleiche Weise bei Rachitis mit dem glandulären Teile der Hypophysis entsprechende Untersuchungen, und fand auf Grund seines - mit 30 proz. Drüsenextrakt der Rindhypophysis gemachten Versuches ausgesprochenen $\mathrm{Ca}$ - und $\mathrm{Mg}$-Verlust.

$$
\begin{gathered}
0,002 \mathrm{MgO} \text { in der ersten Periode } \\
0,308 \mathrm{CaO} " \text { " } \\
-0,015 \mathrm{MgO} \text { im entscheidenden Versuche } \\
-0,102 \mathrm{CaO} "
\end{gathered}
$$

Auf die Frage, ob die Glandula pituitaria in ihrem hinteren 'Teil irgendwelche Wirkung auf die Ausscheidung der Ca-, P- und $\mathrm{Mg}$-Elemente hat, können wir auf Grund unserer Daten mit Bestimmtheit nicht antworten. Wir sahen, dass nach subkutaner Anwendung von täglich $1 \mathrm{cem}$ die Ausscheidung der obigen Elemente nicht in grösserem Masse geschieht. Wir konnten sogar eine Retention geringen Grades beobachten auch noch in dem Falle der Verabreichung des Glanduitrins während 21 Tagen. Wir würden es aber auch schon darum nicht wagen eine so geringe Retention unbedingt auf die Wirkung des Stoffes zurückzuführen, weil wir gerade bei diesen Fällen während der unbeeinflussten Standarddiät eine Retention dèr fraglichen Elemente in grossem Masse finden. Das gleiche sahen wir in jenem Falle, wo die pathologische Entwicklung der Knochen (Chondrodystrophia malacica?) beobachtet werden konnte, und wo nach subkutaner Verabreichung von täglich $1 \mathrm{cem}$ Glanduitrin während 45 Tagen dic Ausscheidungsverhältnisse obiger Elemente untersucht wurden. Wic wir schon erwähnten, kann auch diese lange Serie die Annahme einer zurückhaltenden Wirkung des Glanduitrins auf $\mathrm{Ca}, \mathrm{Mg}$ und $\mathrm{P}$ nicht unterstützen. Und wenn wir jenen Fall betrachten, in dem wir das Verhalten des $\mathrm{Ca}, \mathrm{P}$ und $\mathrm{Mg}$ (II. Fall Fr. Ve.) bei zweifacher Linwirkung des wirkenden Stoffes untersuchten, 
so sehen wir eine ungünstigere Ca-Retention in der Vor- und Nachperiode. In diesem Falle muss auch in Betracht gezogen werden, dass die bei Kranken mit mittelschwerer Osteomalazie bei Ruhe während der Beobachtung gefundene geringe Retention (täglich $13 \mathrm{cg}$ ) nicht die Möglichkeit ausschliesst, dass die Fähigkeit der entsprecbenden Stoffe, das Ca zurückzubchalten, gelitten hat, obzwar die Nachperiode diese Voraussetzung nicht stützt, und eben darum können wir es nicht ausschliessen, dass die ungünstigere Bilanz des $\mathrm{Ca}$ während der Versuchszeit doch in Zusammenhang zu bringen ist mit dem wirkenden Stoffe des hinteren Teiles der Hypophysis. Doch betonen wir wiederholt, dass die Verallgemeinerung eines einzigen Falles gerade bei Osteomalazie nicht richtig ist. Auf unsere Daten müssen wir aber hinweisen, und wir hoffen die Frage zu klären, ob der hintere Teil der Glandula pituitaria in grösserer Dosis die Ca-Retention vermindert oder nicht sowohl unter gesunden Verhältnissen wie bei Osteomalazie? Auch das halten wir der Mühe wert zu erwähnen, dass nach Anwendung der grösseren Dosis eine gesteigerte Ausscheidung des P nicht zu finden war, die Retention des Mg war sogar bedeutend grösser wie in der Beobachtungszeit. Die Verwertung aller dieser Befunde ist bei Osteomalazie darum so schwer, weil wir tatsächlich aus den bis jetzt zur Verfügung stehenden Daten in den durch Medikamente nicht beeinflussten Fällen die Ausscheidungsverhältnisse der verschiedenen Mineralstoffe nicht genau kennen. Und die in Tierversuchen gemachten Erfahrungen können wir für menschliche Osteomalazie nicht für entscheidend halten (Falta und seine Mitarbeiter Mocchi, Francchini: siebe Biedl [Innere Sekretion II. Ausgabe]).

Der Ausbruch des Weltkrieges hat uns an der systematischen Ausführung der Untersuchungen - die wir bei Individuen mit normalem Knochengerüste betreffs der Wirkung der verschiedenen Teile der Hyphophysis auf $\mathrm{Ca}, \mathrm{Mg}$ und $\mathrm{P}$ machen wollten - gehindert. Wir können sagen, dass unsere mit dem glandulären Teile der Hypophysis gemachte Versuchsserie als interessanter Anfang erscheint. Einen so hohen CaVerlust haben wir bei Verabreichung verschiedener Präparate der Hypophysis bisher noch nicht beobachtet; und was wir bei dieser Zusammenfassung noch erwähnen müssen ist der Umstand, dass die Ausscheidung des $\mathbf{P}$ auch bei dieser Gelegenheit weder in gleicher Richtung, noch in gleichem Masse mit der des $\mathrm{Ca}$ geschehen ist.

Hiernach können wir zur Abhandlung der in unserer gegenwärtigen Arbeit eigentlich gestellten Frage übergehen, in welcher Weise der Stoffwechsel des $\mathrm{Ca}, \mathrm{Mg}$ und $\mathrm{P}$ unter dem Einfluss der verschiedenen inneren Drüsen und einzelner chemischer Stoffe bei Osteomalazie modifiziert wird? Zu sämtlichen diesbeziuglichen Untersuchungsdaten hat uns unser I. Fall gedient (Fr. Bá.). Nach Anwendung des hinteren Teiles der Hypophysis hielten wir, wie schon erwähnt, bei der Kranken während 10 Tagen einfach Standarddiät ein, und fanden, dass die tägliche durchschnittliche Retention bei $\mathrm{N}=3,72$, bei $\mathrm{Ca}=0,63$, bei $\mathrm{Mg}=0,08$ und bei $\mathrm{P}=0,7 \mathrm{~g}$ betrug. Wir begannen unsere Untersuchungen am 19.12.1913 mit dem Extrakte des Thymus. Die Budapester Fabrik Richter hat 
uns den entsprechenden Stoff mit grossem Rntyegenkommen zur Verfügung gestelli. Täglich gaben wir $1,1 \mathrm{eem}$ Flïssigkeit subkutarl. Das angewendete Quantum entspricht ungefïlır einem Gramm frischen T'liymusstoffes. Die Kranke klagt während des Versuches über keine unangenehmen Symptome. Dic Periode dauert 14 Tage; die Aufarbeitung wurde in Teilen von je 7 Tagen gemacht. Die Bestimmung der Mineralstoffe graschah in der Thymus- und den ïbrigen Perioden, wio wir schon erwähnten, aus dem periodenweise gemischten, gesamten Urin. (N-Bestimmungen natürlich täglich).

Thy musperiode. (Fr. Bí.)

\begin{tabular}{|c|c|c|c|c|c|c|}
\hline \multirow{2}{*}{$\begin{array}{l}\text { 19. 12. } 1913 \\
\text { bis } \\
\text { 1. 1. } 1914\end{array}$} & \multirow[b]{2}{*}{ Einfuhr } & \multicolumn{3}{|c|}{ Aussoheidung } & \multicolumn{2}{|c|}{ Resultat } \\
\hline & & $\begin{array}{c}\text { Urin } \\
?\end{array}$ & $\begin{array}{c}\text { Fäzes } \\
\text { ? }\end{array}$ & $\begin{array}{c}\text { Zu- } \\
\text { sammen }\end{array}$ & $\begin{array}{c}\text { tud } \\
\text { sammen }\end{array}$ & T'üglich \\
\hline $\mathrm{N}$ & 160,02 & 109,57 & 8,36 & 117,93 & 42,09 & 3,00 \\
\hline $\mathrm{Ca}$ & 19,59 & 0,92 & 8,56 & 9,48 & 10,11 & 0,72 \\
\hline $\mathrm{Mg}$ & 2,65 & 0,63 & 1,34 & 1,97 & 0,68 & 0,048 \\
\hline$P$ & 22,4 & 7,25 & 4,54 & 11,79 & $10,6 \mathrm{I}$ & 0,76 \\
\hline
\end{tabular}

Auch die Thymusperiode zeigte also keine auffallendere Teränderungen der untersuchten Mineralstoffe, besonders wenn wir dic Periode vor dem Thymus zu Grunde legen. Der Nachperiode gegenüber könnten wir schon die geringere Retention des $\mathrm{Ca}, \mathrm{Mg}$ und $\mathrm{P}$. erwähnen. Bieling hat bei rachitischen Kindern Untersuchungen in ähnlicher Richtung gomacht, ebenfalls mit negativem Lrgebnis. Leider steht uns keine solche Untersuchung zur Verfügung, in der wir die Ausscheidung der Mineralstoffe bei grösserem (zweifachem) (Quantum des Thymus untersucht hätten. Die pathologische Thymusfunktion spielt nicht nur in der Pathogenese der Rachitis eine Rolle, sondern auch bei Osteomalazie, und - was im voraus zu sehen war - bezeichnet man den Thymus schon als auslösende Ursache (Scipiades 1917).

Nach der Thymusperiode folgt eine 10 tägige Beobachtungsperiode; damit die Ergebnisse der Einwirkung besser bourteilt werden können. Während der Thymuspause menstruierte die Kranke. Den Urin während der Menstruation haben wir gesondert aufgearbeitet, auch bei den späteren Perioden. In der Tabelle aber (Thymuspause) haben wir die Werte gemeinsam gegeben.

Thy muspause.

\begin{tabular}{|c|c|c|c|c|c|c|}
\hline \multirow{2}{*}{ 2. 1.-11. 1. 1914} & \multirow{2}{*}{ Einfuhr } & \multicolumn{3}{|c|}{ Ausscheidung } & \multicolumn{2}{|c|}{ Resultat } \\
\hline & & $\begin{array}{c}\text { Urin } \\
12610 \mathrm{ccm}\end{array}$ & $\begin{array}{c}\text { Fazes } \\
69 \mathrm{~g}\end{array}$ & $\begin{array}{c}1 / u^{-} \\
\text {sammen }\end{array}$ & $\begin{array}{c}\text { '/u- } \\
\text { sammen }\end{array}$ & 'Täglich \\
\hline $\mathrm{N}$ & 123,48 & 99,54 & 3,43 & 103,38 & 20,10 & 2,01 \\
\hline $\mathrm{Ca}$ & 16,27 & 0,63 & 2,36 & 2,99 & 13,28 & 1,32 \\
\hline $\mathrm{Mg}$ & 2,20 & 0,35 & 0,45 & 0,80 & 1,40 & 0,14 \\
\hline $\mathrm{P}$ & 17,5 & 6,39 & 1,61 & 8,00 & 9,5 & 0,95 \\
\hline
\end{tabular}

Im Verhältnis zur Thymusperiode ist in der Pause dic Retention der Mineralstoffe eine viel günstigere; besonders bexicht sich dies auf" $\mathrm{Ca}$ und $\mathrm{Mg}$. 
Ausser den schon erwähnten Daten von Bieling, welche sich auf Rachitis beziehen, konnten wir gegenwärtig keine auf diese Frage bezügliche Literaturhinweise finden, da sich die Daten von Basch auf Hunde beziehen, bei denen die Exstirpation des Thymus voranging; und an der Hand der. mit den Daten von Basch im Gegensatze stehenden Versuche von Sinnhuber könnten wir unsere negativen Versuche bei Osteomalazie sowieso nicht erklären (Biedl).

Gerne hätten wir in unsere Versuchsserie den Extrakt der Parathyreoidea aufgenommen, doch konnte uns zu unserem grossen Bedauern die Fabrik Richter einen solchen Stoff nicht zur Verfügung stellen; anderswoher konnten wir ihn nicht beschaffen, darum haben wir dic Parathyreoidea von unseren Serien ausgeschlossen; jedoch, wie die Versuche von Bieling zeigen, ist bei der Anwendung dieses Extraktes eine günstige Beeinflussung des $\mathrm{Ca}, \mathrm{Mg}$ und $\mathrm{P}$ zu beobachten.

Die folgende Versuchsserie haben wir mit dem Extrakte der Genitaldrüsen angestellt durch subkutane Verabreichung von täglich $1 \mathrm{ccm}$ Glanduovin und Extr. corp. lutei, welche uns auch von der Fabrik Richter zur Verfügung gestellt worden sind. Auch schon darum haben wir diese Stoffe versucht, weil die heilende Wirkung der Exstirpation dor Ovarien in bestimmten Fällen der Osteomalazie bekannt ist. Seit diescr Entdeckung von Fehling haben viele die pathologische Funktion der Ovarien bei Osteomalazie betont. Auf Grund dieser Auffassung könnten wir als eine Wirkung der obigen Stoffe die ungünstigere Ausscheidung des $\mathrm{Ca}, \mathrm{Mg}$ und $\mathrm{P}$ ansehen. In einem Falle von Neumann folgte auf die ungünstige Ausscheidung des $\mathrm{Ca}, \mathrm{Mg}$ und $\mathrm{P}$ nach Kastration eine sehr starke Retention: $\mathrm{CaO}=-0,99, \mathrm{MgO}=-0,803, \mathrm{P}_{2} \mathrm{O}_{5}=-6,00$ vor der Kastration, $\mathrm{CaO}=+579, \mathrm{MgO}=+4,49, \mathrm{P}_{2} \mathrm{O}_{2}=+6,31$ nach der Rastration.

Glanduovinperiode.

\begin{tabular}{|c|c|c|c|c|c|c|}
\hline \multirow[b]{2}{*}{ 12.--18. 1. 1914} & \multirow[b]{2}{*}{ Einfuhr } & \multicolumn{3}{|c|}{ Ausscheidung } & \multicolumn{2}{|c|}{ Resultat } \\
\hline & & $\begin{array}{c}\text { Urin } \\
8130 \mathrm{ccm}\end{array}$ & $\begin{array}{c}\text { Fäzes } \\
35 \mathrm{~g}\end{array}$ & $\begin{array}{c}Z u- \\
\text { sammen }\end{array}$ & $\begin{array}{c}\mathrm{Zu-} \\
\text { sammen }\end{array}$ & Täglich \\
\hline $\begin{array}{c}\mathrm{N} \\
\mathrm{Ca} \\
\mathrm{Mg} \\
\mathrm{P}\end{array}$ & $\begin{array}{r}82,28 \\
10,70 \\
1,41 \\
11,72\end{array}$ & $\begin{array}{r}51,20 \\
0,40 \\
0,36 \\
3,19\end{array}$ & $\begin{array}{l}2,16 \\
1,15 \\
0,25 \\
0,88\end{array}$ & $\begin{array}{r}53,36 \\
1,50 \\
0,61 \\
4,97\end{array}$ & $\begin{array}{r}28,92 \\
9,15 \\
0,80 \\
7,65\end{array}$ & $\begin{array}{l}4,11 \\
1,307 \\
0,11 \\
1,09\end{array}$ \\
\hline
\end{tabular}

Im Verhältnis zur vorangehenden Periode sind die Retentionswerto um nichts ungünstiger; es besteht bei der angewendeten Dosis keine Spur von gesteigerter $\mathrm{Ca}$, Mg- und P-Ausscheidung. Leider haben wir mit zweifacher Dosis nicht experimentiert, da wir auf die entsprechende Grösse des wirkenden Stoffes erst dann aufmerksam wurden, als wir begonnen hatten die zweifache Dosis des Hypophysenextraktes anzuwenden.

Zwischen den Versuchen mit Glanduovin und mit dem Extr. corp. lutei hielten wir eine 10 tägige Pause, deren Ergebnis die unten folgende Tabelle aufweist. 
Glanduovinpausc.

\begin{tabular}{|c|c|c|c|c|c|c|}
\hline \multirow[b]{2}{*}{ 19.--28. 1. 1914} & \multirow[b]{2}{*}{ Einfuhr } & \multicolumn{3}{|c|}{ Ausscheidung } & \multicolumn{2}{|c|}{ Resultat } \\
\hline & & $\begin{array}{c}\text { Urin } \\
7170 \mathrm{ccm}\end{array}$ & $\begin{array}{l}\text { Fäzes } \\
28 \mathrm{~g}\end{array}$ & $\begin{array}{c}7 \mathrm{Zu} \\
\text { sammen }\end{array}$ & $\begin{array}{c}\text { 7u- } \\
\text { sammen }\end{array}$ & Tïglich \\
\hline $\begin{array}{c}\mathrm{N} \\
\mathrm{Ca} \\
\mathrm{Mg} \\
\mathrm{P}\end{array}$ & $\begin{array}{r}119,88 \\
15,56 \\
2,04 \\
16,96\end{array}$ & $\begin{array}{r}70,21 \\
0,46 \\
0,40 \\
4,95\end{array}$ & $\begin{array}{l}1,67 \\
0,81 \\
0,20 \\
0,75\end{array}$ & $\begin{array}{r}71,88 \\
1,27 \\
0,60 \\
5,70\end{array}$ & $\begin{array}{r}48,00 \\
14,29 \\
1,44 \\
11,26\end{array}$ & $\begin{array}{l}4,8 \\
1,42 \\
0,14 \\
1,12\end{array}$ \\
\hline
\end{tabular}

Die Retention der untersuchten Elemente ist unverändert stark, obzwar wir schon den 80 ten Tag der Stoffwechseluntersuchungen überschritten haben.

Mit dem Extr. corp. lut. haben wir den Versuch während 6 Tagen fortgesetzt, täglich wurde $1,1 \mathrm{ccm}$ subkutan verabreicht (Tabl. Corp. lut.). $1,1 \mathrm{ccm}$ entspricht $1 \mathrm{~g}$ frischem Corp. lut.

Extr. corp. I ut. - Periode.

\begin{tabular}{|c|c|c|c|c|c|c|}
\hline \multirow[b]{2}{*}{ 29. 1.-3. 2. 1914} & \multirow[b]{2}{*}{ Einfuhr } & \multicolumn{3}{|c|}{ Ausscheidung } & \multicolumn{2}{|c|}{ Resultat } \\
\hline & & $\begin{array}{c}\text { Urin } \\
3725 \mathrm{ccm}\end{array}$ & $\begin{array}{l}\text { Fäzes } \\
84 \mathrm{~g}\end{array}$ & $\begin{array}{c}\text { Zu- } \\
\text { sammen }\end{array}$ & $\begin{array}{c}7, u^{-} \\
\text {sammen }\end{array}$ & Täglich \\
\hline $\mathrm{N}$ & 74,58 & 45,03 & 4,36 & 49,39 & 25,19 & 4,19 \\
\hline $\mathrm{Ca}$ & 9,61 & 0,35 & 2,47 & 2,82 & 6,79 & 1,13 \\
\hline $\mathrm{Mg}$ & 1,39 & 0,048 & 0,62 & 0,668 & 0,72 & 0,12 \\
\hline p & 10,57 & 2,71 & 1,91 & 4,62 & 5,95 & 0,99 \\
\hline
\end{tabular}

Wie zu sehen, konnten wir mit dem Extrakte diescr Drüse auch keine Veränderung in den Ausscheidungsverhältnissen des $\mathrm{Ca}, \mathrm{Mg}$ und $\mathrm{P}$ zustande bringen. Wiederum beionen wir auf Grund unserer bisherigen Erfahrungen, dass vielleicht die Dosis zu klein war.

Nach dieser Periode schalten wir eine 7 tägige Pause ein, inzwischen tritt wieder Menstruation auf. Bei der Aufarbeitung des Urins verfahren wir nach den schon erwähnten Regeln. (Menstruation am 5. und 6. 2.)

Extr. corp. Iut.-Pause.

\begin{tabular}{|c|c|c|c|c|c|c|}
\hline \multirow[b]{2}{*}{ 4.--10. 2. 1914} & \multirow[b]{2}{*}{ Einfuhr } & \multicolumn{3}{|c|}{ Ausscheidung } & \multicolumn{2}{|c|}{ Resultat } \\
\hline & & $\begin{array}{c}\text { Urin } \\
3930 \mathrm{ccm}\end{array}$ & $\begin{array}{c}\text { Fäzes } \\
66 \mathrm{~g}\end{array}$ & $\begin{array}{c}Z \mathrm{Zu}- \\
\text { sanmen }\end{array}$ & $\begin{array}{c}\mathrm{Zu} \cdot \\
\text { sammen }\end{array}$ & Täglinh \\
\hline $\mathrm{N}$ & 87,78 & 54,08 & 4,72 & 58,80 & 29,98 & 4,14 \\
\hline $\mathrm{Ca}$ & 11,17 & 0,45 & 2,32 & 2,77 & 8,40 & 1,20 \\
\hline $\mathrm{Mg}$ & 1,68 & $0,196(?)$ & 0,47 & 0,66 & 1,02 & 0,14 \\
\hline $\mathrm{P}$ & 12,60 & 7,71 & 1,34 & 9,15 & 3,45 & 0,49 \\
\hline
\end{tabular}

Die im Verhältnis grosse Ausscheidung des $\mathrm{P}$ im Urin ist das Ergebnis der Menstruation.

Betrachten wir unsere weiteren Untersuchungsdaten, welche wir mit dem wirkenden Stoffe der Nebenniere gemacht haben. Es ist bekannt, dass in der Pathogenese, ja auch in der Aetiologie und Therapie der Osteomalazie auch der Hypofunktion der Nebenniere eine Rolle zugesprochen ist, auf Grund einer Annahme von Bossi. Nach ihm wäre die Ursache der Osteomalazie das Ausfallen oder das Sinken der Neben- 
nierenfunktion. In der Pathogenese der Rachitis hat Stöltzner schon früher die Bedeutung der Nebenniere betont. Und zwar sieht er die Bedeutung der Funktion der pathologischen Nebenniere darin, dass das rachitische Osteoidgewebe - hinsichtlich der Fähigkeit $\mathrm{Ca}$ aufnehmen zu können - auf chemischem Wege eine Veränderung erfährt.

Wi haben unserer Kranken Adrenalin (Clin.) subkutan verabreicht, und zwar am ersten Tage $1 \mathrm{ccm}(1 / 2 \mathrm{mg})$, während der übrigen 9 Tage täglich $1 / 4 \mathrm{mg}$.

Adrenalinperiode.

\begin{tabular}{|c|c|c|c|c|c|c|}
\hline \multirow[b]{2}{*}{$11,-20.2 .1914$} & \multirow[b]{2}{*}{ Einfuhr } & \multicolumn{3}{|c|}{ Ausscheidung } & \multicolumn{2}{|c|}{ Resultat } \\
\hline & & $\begin{array}{c}\text { Urin } \\
5250 \mathrm{ccm}\end{array}$ & $\begin{array}{l}\text { Fäzes } \\
152 \mathrm{~g}\end{array}$ & $\begin{array}{c}\mathrm{Zu}- \\
\text { sammen }\end{array}$ & $\begin{array}{c}\mathrm{Zu-} \\
\text { sammen }\end{array}$ & Täglich \\
\hline $\begin{array}{c}\mathrm{N} \\
\mathrm{Ca} \\
\mathrm{Mg} \\
\mathrm{P}\end{array}$ & $\begin{array}{r}123,28 \\
15,14 \\
2,32 \\
17,55\end{array}$ & $\begin{array}{r}80,98 \\
0,94 \\
0,43 \\
5,88\end{array}$ & $\begin{array}{l}8,16 \\
7,22 \\
1,32 \\
3,31\end{array}$ & $\begin{array}{r}89,14 \\
8,16 \\
1,75 \\
9,19\end{array}$ & $\begin{array}{r}34,14 \\
6,98 \\
0,57 \\
8,36\end{array}$ & $\begin{array}{l}3,41 \\
0,698 \\
0,057 \\
0,836\end{array}$ \\
\hline
\end{tabular}

In dieser Periode fällt es auf, dass die Ca-Retention eine nicht so grosse ist wie in den vorigen Perioden, wir können sogar sagen, dass wir während der ganzen Versuchsserie jetzt den niedrigsten Wert der Ca-Retention sehen. Wenn wir absehen von unseren weiteren Daten, so könnten wir diese im Verhältnis stärkere Ca-Ausscheidung dem reichlicheren und öfteren Stuhlgange zuschreiben, der in dieser Periode vorhanden war; andererseits müssen wir schon jetzt den Umstand erwähnen, dass mit dem Urin täglich im Durchschnitte $9 \mathrm{cg} \mathrm{Ca}$ ausgeschieden worden sind. Und einen solchen Wert haben wir in diesem Falle bis jetzt noch nicht beobachtet. Die $\mathrm{N}$-Ausscheidung zeigt nichts besonderes. Es ist zu erwähnen, dass mit der geringeren Ca-Retention das Verhalten des $\mathrm{Mg}$ auch parallel geht. Der Wert dieser Periode ist mehr oder weniger gleich jenem, welchen wir in der Thymusperiode gefunden haben; nur während wir in der Thymuspause die gewohnte hochgradige Retention der fraglichen Elemente beobachteten, sind in der 9 Tage dauernden Adrenalinpause die Ausscheidungswerte ganz andere.

Adrenalinpause.

\begin{tabular}{|c|c|c|c|c|c|c|}
\hline \multirow[b]{2}{*}{ 21. 2.-1. 3. 1914} & \multirow[b]{2}{*}{ Einfuhr } & \multicolumn{3}{|c|}{ Ausscheidung } & \multicolumn{2}{|c|}{ Resultat } \\
\hline & & $\begin{array}{c}\text { Urin } \\
6045 \mathrm{ccm}\end{array}$ & $\begin{array}{l}\text { Fäzes } \\
145 \mathrm{~g}\end{array}$ & $\begin{array}{c}\mathrm{Zu}- \\
\text { sammen }\end{array}$ & $\begin{array}{c}\mathrm{Zu}- \\
\text { sammen }\end{array}$ & Täglich \\
\hline $\begin{array}{c}\mathrm{N} \\
\mathrm{Ca} \\
\mathrm{Mg} \\
\mathrm{P}\end{array}$ & $\begin{array}{r}113,54 \\
14,20 \\
2,08 \\
15,98\end{array}$ & $\begin{array}{r}93,27 \\
0,67 \\
0,30 \\
7,46\end{array}$ & $\begin{array}{r}5,28 \\
11,67 \\
1,15 \\
4,11\end{array}$ & $\begin{array}{r}98,55 \\
12,34 \\
1,45 \\
11,57\end{array}$ & $\begin{array}{r}14,99 \\
1,86 \\
0,63 \\
4,41\end{array}$ & $\begin{array}{l}1,66 \\
0,21 \\
0,07 \\
0,49\end{array}$ \\
\hline
\end{tabular}

Die N-Retention ist verhältnismässig geringer, aber die kleinere Ca-Retention ist doch auffallend, während der geringere Wert des $\mathrm{P}$ auf Grund der übrigen Daten nicht überraschend ist. Diese ungünstigere Retention können wir schon mit dem täglich auftretenden breiigen Stuhle in Zusammenhang bringen, weil die Kranke während unserer bisherigen Untersuchungen so reichlichen, wässerigen Stuhl bis 
jetzt noch nicht entleert hatte; obzwar wir in unseren Tabellen orwähnten, dass während der Glanduovinperiode der Stuhl teils breiig, teils flüssig ist, ist troizdem das Quantum des mit den Fäzes entfernten Ca viel geringer.

Mit der Adrenalinperiode haben wir eigentlich jenen T'eil unserer Untersuchungen - in denen wir mit den eventuell wirkenden Stoffen der einzelnen inneren Drüsen die Ausscheidungsverhältnisse des $\mathrm{Ca}, \mathrm{Mg}$ und $\mathrm{P}$ crforschten - beendigt und gaben nach der 9 tägigen Adrenalinpause der Kranken per os Calcium lacticum täglich $4,0 \mathrm{~g}(0,51 \mathrm{~g} \mathrm{Ca})$.

Calcium lacticum-Periode.

\begin{tabular}{|c|c|c|c|c|c|c|}
\hline \multirow{2}{*}{$\begin{array}{c}2 .-8.3 . \\
1914\end{array}$} & \multirow[b]{2}{*}{ E in $\mathrm{fur}$} & \multicolumn{3}{|c|}{ Ausscheidung } & \multicolumn{2}{|c|}{ Resultat } \\
\hline & & $\begin{array}{c}\text { Urin } \\
4900 \mathrm{ccm}\end{array}$ & $\begin{array}{l}\text { Fäzes } \\
137 \mathrm{~g}\end{array}$ & $\begin{array}{c}\mathrm{Zu-} \\
\text { sammen }\end{array}$ & $\begin{array}{c}\mathrm{Zu-} \\
\text { sammen }\end{array}$ & Täglich \\
\hline $\begin{array}{c}\mathrm{N} \\
\mathrm{Ca} \\
\mathrm{Mg} \\
\mathrm{P}\end{array}$ & $\begin{array}{c}88,62 \\
3,63+11,03=1466 \\
1,62 \\
12,49\end{array}$ & $\begin{array}{r}91,96 \\
0,86 \\
0,16 \\
5,14\end{array}$ & $\begin{array}{r}5,89 \\
10,97 \\
1,57 \\
4,86\end{array}$ & $\begin{array}{r}97,85 \\
11,83 \\
1,73 \\
10,00\end{array}$ & $\begin{array}{r}-9,23 \\
2,83 \\
-0,11 \\
2,49\end{array}$ & $\begin{array}{l}-1,31 \\
0,404 \\
-0,015 \\
+0,35\end{array}$ \\
\hline
\end{tabular}

Während der zwei letzten Tagen der Calc. lact.-Periode tritt Menstruation auf, damit liegt ein störender Umstand vor, auch der Stuhl hat noch immer eine mehr breiartige Konsistenz, aber in kleinerem Masse wie in der vorhergehenden Periode; allerdings sehen wir bei der Kranken jetzt zum erstenmal ein Plus der $\mathrm{N}$-Ausscheidung, ebenso auch beim $\mathrm{Mg}$. Auch die Ca-Ausscheidung ist ungünstig, da, wenn wir den täglich zugeführten Wert des Calcium lacticum $(4 \mathrm{~g})$ nicht in Betracht ziehen würden, die Periode mit 80 ctg Ca-Verlust geendet hätte.

Mit Aussetzen des Calcium lacticum halten wir eine 8 tägige Pausc ein. Am ersten Tag ist noch Menstruation vorhanden. Der Stuhl ist teils geformt, teils breiig.

Am 14. 3. treten heftige Unterleibsschmerzen auf, einmal Erbrechen. Ther.: 10 Tropfen Morphium von 1 proz. Lösung. Am 15. 3. fühlt sich die Patientin leichter.

Calcium lacticum-Pause.

\begin{tabular}{|c|c|c|c|c|c|c|}
\hline \multirow{2}{*}{$8 .-16.3 .1914$} & \multirow{2}{*}{ Einfuhr } & \multicolumn{3}{|c|}{ Ausscheidung } & \multicolumn{2}{|c|}{ Resultat } \\
\hline & & $\begin{array}{c}\text { Urin } \\
7205 \mathrm{ccm}\end{array}$ & $\begin{array}{l}\text { Fäzes } \\
100 \mathrm{~g}\end{array}$ & $\begin{array}{c}\text { Zu- } \\
\text { sammen }\end{array}$ & $\begin{array}{c}\text { Zu- } \\
\text { sammen }\end{array}$ & Täglich \\
\hline $\begin{array}{c}\mathrm{N} \\
\mathrm{Ca} \\
\mathrm{Mg} \\
\mathrm{P}\end{array}$ & $\begin{array}{r}100,10 \\
12,50 \\
1,81 \\
14,20\end{array}$ & $\begin{array}{r}80,35 \\
0,84 \\
0,49 \\
5,95\end{array}$ & $\begin{array}{r}4,09 \\
10,48 \\
1,39 \\
4,77\end{array}$ & $\begin{array}{r}84,44 \\
11,32 \\
1,88 \\
10,72\end{array}$ & $\begin{array}{r}15,66 \\
1,18 \\
-\quad 0,07 \\
3,48\end{array}$ & $\begin{array}{c}1,85 \\
0,14 \\
-0,007 \\
0,43\end{array}$ \\
\hline
\end{tabular}

Auch diesmal sehen wir $\mathrm{Mg}$-Verlust; die $\mathrm{Ca}$-Retention ist klein. Wegen der störenden Nebenumstände wäre es schwer, die Daten im Zusammenhange mit dem zugeführten Ca-Salze zu verwerten. Auf Grund der lange Zeit dauernden Mineralstoffwechseluntersuchungen ist Vorsicht beim Verwerten der Daten anzuraten. 
Daten zur Wirkung der Extrakto oiniger inmerer Drüsen bei Osteomalazie. 121

In der folgenden Serie bekommt unserc Kranke Phosphor. Täglich verabreichen wir $2 \mathrm{mg}$ in 0 l. arnygd. dulcium-Lösung während 7 Tagen; das Allgemeinbefinden ist ungestört gut.

Phosphorperiode.

\begin{tabular}{|c|c|c|c|c|c|c|}
\hline \multirow{2}{*}{$\begin{array}{c}17 .-23.3 \\
1914\end{array}$} & \multirow{2}{*}{ Einfuhr } & \multicolumn{3}{|c|}{ A usscheidung } & \multicolumn{2}{|c|}{ Resultat } \\
\hline & & $\begin{array}{c}\text { Urin } \\
9595 \mathrm{ccm}\end{array}$ & $\begin{array}{l}\text { Fäzes } \\
129 \mathrm{~g}\end{array}$ & $\begin{array}{c}\mathrm{Zu}- \\
\text { sammer }\end{array}$ & $\begin{array}{c}\text { Zu- } \\
\text { sammen }\end{array}$ & Täglich \\
\hline $\begin{array}{c}\mathrm{N} \\
\mathrm{Ca} \\
\mathrm{Mg} \\
\mathrm{P}\end{array}$ & $\begin{array}{c}89,74 \\
11,33 \\
1,68 \\
12,85+0,014 \mathrm{~g} \mathrm{P}\end{array}$ & $\begin{array}{r}87,31 \\
0,30 \\
0,50 \\
7,26\end{array}$ & $\begin{array}{r}5,54 \\
11,44 \\
1,45 \\
4,23\end{array}$ & $\begin{array}{r}92,85 \\
11,74 \\
1,95 \\
11,49\end{array}$ & $\begin{array}{l}-3,11 \\
-0,41 \\
-0,27 \\
+1,36\end{array}$ & $\begin{array}{l}-0,44 \\
-0,05 \\
-0,039 \\
+0,19\end{array}$ \\
\hline
\end{tabular}

In dieser Periode haben wir keine störenden Nebenumstände und doch finden wir eine ungünstige Ausscheidung der untersuchten Stoffe. Mit Ausnahme des $\mathrm{P}$ wird diese Serie mit negativem Ergebnis abgeschlossen; selbst wenn wir die 7 Tage lang dauernde Nachperiode betrachten, wo wir uns ebenfalls auf keinen störenden Umstand beziehen können, sehen wir einen Verlust an Ca.

Phosphorpausc.

\begin{tabular}{|c|c|c|c|c|c|c|}
\hline \multirow[b]{2}{*}{$24 .-30.3 .1914$} & \multirow{2}{*}{ Einfuhr } & \multicolumn{3}{|c|}{ Ausscheidung } & \multicolumn{2}{|c|}{ Resulvat } \\
\hline & & $\begin{array}{c}\text { Urin } \\
8945 \mathrm{ccm}\end{array}$ & $\begin{array}{l}\text { Fäzes } \\
107 \mathrm{~g}\end{array}$ & $\begin{array}{c}Z u- \\
\text { sammen }\end{array}$ & $\begin{array}{c}\mathrm{Zu}- \\
\text { sammen }\end{array}$ & Täglich \\
\hline $\mathrm{N}$ & 89,74 & 78,50 & 4,40 & 82,90 & $+6,84$ & 0,97 \\
\hline $\mathrm{Ca}$ & 11,33 & 0,58 & 11,21 & 11,79 & $-0,46$ & $-0,06$ \\
\hline $\mathrm{Mg}$ & 1,68 & 0,30 & 0,90 & 1,20 & $+0,48$ & 0,068 \\
\hline $\mathrm{P}^{\mathrm{P}}$ & 12,71 & 7,01 & 3,91 & 10,92 & $+1,79$ & $+0,25$ \\
\hline
\end{tabular}

Deutlich sehen wir aber beim Mg Retention, ebenso beim $N$ und $P$. Allerdings möchten wir die P-Wirkung bei Osteomalazic nicht mit einem solchen Befund beweisen.

In der letzten Versuchsphase geben wir der Kranken Tricalciumphosphat in einer Dosis von täglich $5 \mathrm{~g}(1,94 \mathrm{~g} \mathrm{Ca}$ und $1,0 \mathrm{~g} \mathrm{P})$. Die Periode dauerte 6 Tage.

Tricalciumphosphat-Tabelle.

\begin{tabular}{|c|c|c|c|c|c|c|}
\hline \multirow{2}{*}{$\begin{array}{l}\text { 31. } 3 .-5.4 \text {. } \\
1914\end{array}$} & \multirow{2}{*}{ Einfuhr } & \multicolumn{3}{|c|}{ A usscheidung } & \multicolumn{2}{|c|}{ Resultat } \\
\hline & & $\begin{array}{c}\text { Urin } \\
7560 \mathrm{~cm}\end{array}$ & $\begin{array}{l}\text { Fäzes } \\
161 \mathrm{~g}\end{array}$ & $\begin{array}{c}\mathrm{Zu-} \\
\text { sammen }\end{array}$ & $\begin{array}{c}\mathrm{Zu} \mathbf{*}^{-} \\
\text {sammen }\end{array}$ & Täglich \\
\hline $\mathrm{N}$ & $\begin{array}{l}80,36 \\
22,33\end{array}$ & 70,59 & 6,42 & 77,01 & 3,35 & $+0,55$ \\
\hline $\mathrm{Ca}$ & $\overline{10,69+11,64}$ & 0,44 & 13,83 & 14,27 & 8,06 & $+1,34$ \\
\hline $\mathrm{Mg}$ & $\begin{array}{c}1,54 \\
19,17\end{array}$ & 0,35 & 1,52 & 1,87 & $-0,35$ & $-0,05$ \\
\hline $\mathbf{P}$ & $11,57+7,6$ & 8,41 & 5,02 & 13,43 & 4,14 & $+0,69$ \\
\hline
\end{tabular}

Die Ausscheidung des Mg-Elementes übertrifft die Einfuhr; scheinbar ist sie wieder negativ im Gegensatze zu den übrigen Elementen. Doch wenn wir die Vorhauptperiode betrachten, könuen wir eine negative 
Bilanz des $\mathrm{Ca}$ und eine positive des $\mathrm{Mg}$ sehen. In dieser Periode aber, wo das Quantum des künstlich zugeführten Ca sehr gross ist, ist zwar die Bilanz des Ca positiv, jedoch nur dann, wenn wir auch den durch Ca-Phosphat zugeführten Wert in Rechnung nehmen. Dasselbe können wir auch über den $\mathrm{P}$ sagen. Das negative Ergebnis des $\mathrm{Mg}$ zeigt also eigentlich das wirkliche Bild; dass nämlich für die Retention des $\mathrm{Ca}, \mathrm{Mg}$ und $\mathrm{P}$ die Zeitphase nicht günstig ist.

Endlich erwähnen wir die letzte Periode, welche nur 4 Tage dauerte, ausserdem fehlen uns vom Urin die $\mathrm{Ca}-\mathrm{Mg}$ - und P-Werte, infolgedessen können unsere Daten quantitativ nicht in Rechnung kommen.

Tricalicumphosphat-Pause.

\begin{tabular}{|c|c|c|c|c|c|c|}
\hline \multirow[b]{2}{*}{$6 .-9.4 .1914$} & \multirow[b]{2}{*}{ Einfuhr } & \multicolumn{3}{|c|}{ Aussehoidung } & \multicolumn{2}{|c|}{$R e s u l t a t$} \\
\hline & & $\begin{array}{l}\text { Urin } \\
3195 \mathrm{cem}\end{array}$ & $\begin{array}{l}\text { Fäzes } \\
81 \mathrm{~g}\end{array}$ & $\begin{array}{c}\text { Zu- } \\
\text { sammen }\end{array}$ & $\begin{array}{c}\mathrm{Zu}- \\
\text { sammen }\end{array}$ & Täglich \\
\hline $\begin{array}{c}\mathrm{N} \\
\mathrm{Ca} \\
\mathrm{Mg} \\
\mathrm{P}\end{array}$ & $\begin{array}{r}82,22 \\
4,88 \\
0,72 \\
5,40\end{array}$ & $\begin{array}{l}33,27 \\
- \\
-\end{array}$ & $\begin{array}{l}3,70 \\
5,84 \\
0,77 \\
2,59\end{array}$ & $\begin{array}{c}36,90 \\
- \\
-\end{array}$ & $\begin{array}{c}1,32 \\
-0,96(?) \\
-0,05(?) \\
-\end{array}$ & $\begin{array}{c}+0,33 \\
-0,24(?) \\
-0,01(?) \\
-\end{array}$ \\
\hline
\end{tabular}

Obzwar wir die Daten des Urins wegen äusserer Umstände nicht bestimmen konnten, können wir doch sagen, dass in der Ausscheidung der Ca- und $\mathrm{Mg}$-Elemente wieder eine Störung gesehen ist und zwar in bedeutendem Grade; können wir die Daten des Urins angeben, so würde das beim $\mathrm{Mg}$ ein ziemlich bedeutendes Plus bedeuten. Die N-Retention ist gering. Und was den $\mathrm{P}$ anbelangt, so läge, wenn wir nur auf Grund der vorhergehenden Untersuchungen für den P-Inhalt des Urins einen Wert von 1 pCt. annehmen würden, auch die Ausscheidung dieses Stoffes dem eingeführten Quantum gegenüber ungünstig.

Wir haben die Versuche nicht weiter fortgesetzt, die Kranke blieb aber unter weiterer klinischer Beobachtung, zeitweise (in den Monaten Mai und Juni) hat sie wiederholt Pituitrin bekommen, dann wieder Calcium lacticum. Ihr Allgemeinbefinden besserte sich. Ihre Schmerzen haben nachgelassen, und nach fast einjährigem Aufenthalt verliess die Kranke die Klinik bedeutender gebessert.

Nach Aufzählung unserer Daten fragt es sich jetzt, ob wir auf unsere, bei Beginn des Versuches erwähnte Annahme - dass bei Osteomalazie verschiedene innere Drüsen, im Aufbau der Knochen Verschiebungen bewirken - eine Antwort geben können oder nicht? Unser diesbezüglicher Versuch bezieht sich eigentlich nur auf einen Fall. Unsere übrigen Untersuchungen weisen auf jene Daten hin, welche sich auf die Wirkung des hinteren und teils des glandulären Teiles der Hypophysis beziehen; und von diesen haben wir auch schon anderen Ortes Erwähnung getan. Bei der endgültigen Folgerung ist aber die die Verwendung dieser Daten berechtigt.

Die Folgerung aus Stoffwechseluntersuchungen ist sehr schwer; abgesehen von Fehlern, die sich bei Versuchen ergeben, ist Folgerung von Daten aus einem einzigen Ealle nicht empfehlenswert. Auch das müssen wir 
in Betracht zichen, dass sich unsere gegenwärtigen Daten auf Osteomalazie beziehen. Auch die Krankheit selbst ist nicht verbreitet, es ist daher natürlich, dass die Stoffwechseluntersuchungen im Verhältnisse zur Seltenheit der Krankheit selten gemacht worden sind. Jedoch jene grosse Masse der Daten, welche für die Rachitis in der Weltliteratur zur Verfügung stehen, wird es selbst nach langer Zeit schwer sein bei Osteomalazie zusammen zu bringen. Aus den bei Rachitis in so grosser Zahl gemachten Stoffwechseluntersuchungen aber ist das Verhalten der Stoffe, welche das Knochengerüst aufbauen, mit Ausnahme des $\mathrm{Ca}$, das des $\mathrm{Mg}, \mathrm{P}$ und $\mathrm{CO}_{2}$ noch nicht bekannt. Das Verhaiten des ersteren ist kaum bekannt, betreffs des letzteren stehen uns systematische Untersuchungen überhaupt nicht zur Verfügung. Auch sogar das Verhalten des P-Elementes ist in dem Masse nicht bekannt, wie das des Ca. Bei Rachitis wird also in der Zukunft unter den Mineralstoffen das Verhalten des $\mathrm{P}$ und $\mathrm{CO}_{2}$, sogar das des $M g$ sowohl in den unbeeinflussten, als in den auf verschiedene Weise beeinflussten Fällen Gegenstand der Forschung bilden. Bei Osteomalazie stehen wir auf diesem Gebiete noch ganz im Anfang, und unsere gegenwärtigen Datenkenntnisse haben eigentlich noch nicht über den Wert der Datensammlung geführt. Aber auf Grund der Benutzung von den bei Rachitis gewonnenen Daten ist eine bestimmte Richtung der Untersuchungen bei Osteomalazie bedeutend leichter geworden. Die Orientierungsversuche müssen in gleicher Richtung vor sich gehen, wie bei Rachitis; und abgesehen von dem grossen Unterschied, welcher zwisehen einem in Entwickelung stehenden und dem schon entwickelten, in seiner Entwickelung stehen gebliebenen Organismus auch im Stoffwechselleben besteht, meinen wir, dass zur Beurteilung des Verhaltens der im Aufbau der Knochen mitwirkenden Stoffe dic Untersuchungen gleicher Richtung bei Osteomalazic nur vorteilhaft sein werden.

Den Wert unserer Daten zu überschätzen wäre also ein Fehler. Abgesehen davon, dass wir unsere Erfahrungen nur in einem Falle sammelten, waren unsere Untersuchungen gerade in der Entwickelung, als uns das Auftreten der Kriegsverhältnisse im Aufbaue unserer Arbeit hinderte, so dass auch noch jener Teil unserer Untersuchungen lückenhaft blieb, in welchem wir die Wirkungsweise der Hypophysis untersuchten, obzwar die Wichtigkeit der Qualität und Quantität der wirkenden Stoffe, und das gęeignete Auswählen der Fälle beim Verwerten unserer Versuche schon damals im Vordergrunde standen.

Unsere Daten ganz zu vernachlässigen wäre andererseits nicht zweckmässig, weil wir sie in einem Falle von Osteomalazie gewonnen haben, wobei wir 149 Tage hindurch die Beobachtungen machten. Eine so lange dauernde Stoffwechseluntersuchung hinsichtlich der knochenaufbauenden Stoffe ist gerade bei Osteomalazie ergänzend. Wir glauben nicht, dass wir bei Osteomalazie vor Auftreten der Krankheit diese Daten planmässig untersuchen können. Hier würde dem Untersucher nur der Zufall helfen, und darum kann uns nur teilweise der Vorwurf treffen, dass wir unsere Untersuchungen bei einer Osteomalaziekranken machten, bei der das Verhalten der Mineralstoffe schon den Beginn der Besserung zeigte. Unsere Kranke zeigte bei Beginn der Untersuchung einen Rückfall der chronischen 
mittelschweren Osteomalazic. (Sio konnte nicht gehen, dio Knochen waren schr empfindlich usw.) Und so hat uns die schon in der Beobachtungsperiode gefundene günstige Retention der gesuchten Elemente nicht unbedingt beweisen können, dass tatsächlich eine längere Zeit dauernde günstige Phase vor uns stehen wird.

Als wir die Versuche bei unserer Kranken mit den Extrakten der inneren Drüsen begonnen hatten, sahen wir neben hoher N-Retention auch bei den übrigen Elementen eine Zurückhaltung; und wir sprechen es diesem I imstande zu, dass wir im angewendeten Quantum während der 21 Tage lang dauernden Glanduitrinperiode keine so hochgradige $\Lambda$ bweichung gefunden haben, aus der unsere Folgerung in irgend einer Richtung bestimmt wäre. Die Retention ist in der Versuchsphase zur Genüge zu beobachten; sogar wenn wir die 21 tägige Beobachtungsperiode betrachten, sind dic Daten im Verhältnisse zur Vorperiode günstiger bei allen vier Elementen; als Beweis könnten wir sogar auch das erwähnen, dass in der nach der Glanduitrinperiode folgenden Pause die Retentionswerte bei allen vier Elementen niedriger sind, und zwar abgesehen vom $P$ haben wir noch niedrigere Werte bekommen, wie die in der Grundperiode beobachteten sind; doch trotzdem hüten wir uns vor der Aeusserung, dass die stärkere Retention der Elemente in der Glanduitrinperiode durch Wirkung des angewendeten Stofles erfolgt sci. (Siehe die Prozenttabelle.) Bei der Thymusperiode können wir uns ähnlicherweise über die Wirkung des angewendeten Stoffes nicht äussern. Dic Retention ist im Verhältnis zur Grundperiode etwas schlechter (ausgenommen das P), der vorangehenden Periode gegenüber ist hauptsächlich die $\mathrm{Mg}$-Retention gesunken. Nach dem Weglassen des T'hymusstoffes ist die Retention abgesehen vom $\mathrm{N}$ bedentend günstiger, was das $\mathrm{Ca}$ und $\mathrm{Mg}$ betrifft, so ist die Retention sogar ungewöhnlich gross. Nach Anwendung des wirkenden Stoffes der Ovarien ist die Retention wieder günstiger (seltener Stuhlgang, Gewicht der trockenen Fäzes 35 und $28 \mathrm{~g}$ [sehr wenig]). Später in der Adrenalinperiode sehen wir die Retention des $\mathrm{Ca}$ und $\mathrm{Mg}$ in woniger günstigem lichte, dies setzt sich hauptsächlich für das Ca in der Pause fort, wo zugleich auch die Eiweissretention etwas ungünstiger geworden ist.

Retention in Prozent der Aufnahme.

\begin{tabular}{|c|c|c|c|c|c|c|c|c|c|c|c|}
\hline & 宫总 & 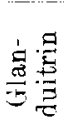 & Pause & 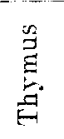 & Pause & 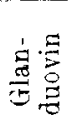 & Pause & 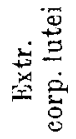 & Pause & 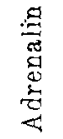 & Pause \\
\hline $\mathrm{N}$ & 36,5 & 39,6 & 31,1 & 26,2 & 16,2 & 34,8 & $\cdot 40,0$ & 39,3 & 33,0 & 34,8 & 13,2 \\
\hline$i_{A}$ & 6,79 & 75,7 & 40,0 & 51,6 & 81,6 & 85,6 & 91,8 & 70,6 & 75,2 & 46,0 & 13,1 \\
\hline $\mathrm{Mg}$ & 50,1 & 68,5 & 45,8 & 25,6 & 66,6 & $56, \pi$ & 70,5 & 51,8 & 60,7 & 24,5 & 30,2 \\
\hline$P^{b}$ & 24,4 & 61,4 & 41,2 & 47,3 & 54,2 & 65,2 & 66,3 & 56,2 & 27,3 & 47,6 & 59,9 \\
\hline
\end{tabular}

Wenn wir unsere Untersuchungen nicht weitor fortsetzen, so sehen wir doch aus diesen Daten, dass bei unserer Kranken eine Retention der untersuchten Stoffe mit grösseren oder kleincren Schwankungen vorliegt. Jene Frage, ob diese klcineren Veränderungen mit den angewendeten Stoffen in Zusammenhang zu bringen sind, können wir, wie 
schon erwähnt, nicht beantworten. Wir sehen, dass wir in keiner Serie eine ungünstige Bilanz erhalten. Können wir daher sagen, dass einer oder der andere der benutzten inneren Drüsenstoffe (T'hymus, Adrenalin) die Ausscheidung der untersuchten Elemente ungünstig beeinflusst hätte? Wenn wir nur diese Tabelle zugrunde legen, dann auf keinen Fall. Mit demselben Rechte könnten wir sagen, dass die Wirkungsweise nicht zur Geltung kommen konnte, weil der wirkende Stoff eventuell zu gering war oder dass die Anwendungsweise nicht zweckdienlich war (vielleicht hätten frische Drüsenextrakte angewendet werden müssen). Und endlich könnten wir auch das sagen, dass unsere Kranke bis zum 90. Tage der Beobachtung in einer solchen Periode war, wo sie die knochenaufbauenden Stoffe so stark zurückhielt, dass wir diesen Ca-, Mg- und P-Hunger des Organismus mit den angewendeten milden Einwirkungen, ja sogar vielleicht mit energischeren Mitteln auch nicht hätteu modifizieren können. Doch all dies sind nur Annahmen, die noch zu beweisen sind. Keine unserer Annahme können wir mit positiven Daten beweisen, denn auch unsere mit dem verschiedenen Quantum der Hypophysis gemachten Untersuchungen müssen noch ergänzt werden, ohne die mit Thymusextrakt, Extr. corp. lutei usw. angefertigten Untersuchungen erwähnt zu baben.

Während der Anwendung des Adrenalins tritt ein reichlicherer und häufigerer Stuhlgang auf und auch das Gewicht der trockenen Fäzes ist viel grösser als z. B. in der Glanduovinperiode, mit der im Zusammenhang auch die absoluten ausgeschiedenen Werle grösser sind. Wenn uns also spätere Untersuchungen nicht zur Verfügung stehen würden, so wären wir geneigt, das Sinken der Retention nur auf diesen Umstand zurückzuführen.

Wenn wir die über diese Daten handelnde zusammenfassende Tabelle betrachten, so sehen wir, dass der Organismus neben der bedeutenden N-Retention auch von den Mineralstoffen reichlich zurückbehalten hat.

\begin{tabular}{|c|c|c|c|c|c|}
\hline \multirow{2}{*}{$\begin{array}{c}\text { Dauer der Versuche } \\
110 \text { Tage } \\
\text { 11. 10. } 1913 \text { bis } \\
\text { 1. 2. } 1914\end{array}$} & \multirow{2}{*}{$\begin{array}{l}\text { Gesamt- } \\
\text { einfuhr }\end{array}$} & \multicolumn{3}{|c|}{ A us schcidung } & \multirow{2}{*}{ Resultat } \\
\hline & & Urin & Fäzes & $\begin{array}{c}\text { Zu- } \\
\text { sammen }\end{array}$ & \\
\hline $\begin{array}{c}\mathrm{N} \\
\mathrm{Ca} \\
\mathrm{Mg} \\
\mathrm{P}\end{array}$ & $\begin{array}{r}1330,59 \\
174,64 \\
22,91 \\
189,55\end{array}$ & $\begin{array}{r}816,91 \\
6,01 \\
3,37 \\
63,23\end{array}$ & $\begin{array}{r}50,69 \\
55,40 \\
7,71 \\
35,12\end{array}$ & $\begin{array}{r}\$ 67,60 \\
61,41 \\
11,08 \\
98,35\end{array}$ & $\begin{array}{l}+452,99 \\
+113,23 \\
+\quad 11,83 \\
+\quad 91,20\end{array}$ \\
\hline
\end{tabular}

Die Retention von $113 \mathrm{~g}$ Ca während 110 Tagen weist auf das grosse Ca-Bedürfnis des Organismus hin. Und das zurückgehaltene $\mathrm{P}$ würde reichlich dem Quantum der im Knochen vorkommenden komplexen Kalziumverbindung entsprechen. Bisher haben wir in diesen Serien gefunden, dass die Retention des $\mathrm{Mg}$ mehr oder weniger im Verhältnis zum $\mathrm{Ca}$ geschieht. (Die Periode der Adrenalinpause ist eine Ausnahme.)

Die während der Einwirkung der Extrakte der inneren Drüsen gewonnenen Daten haben wir ganz besonders bei jenen Untersuchungen betrachtet, welche wir mit den Ca-Salzen und mit dem $\mathrm{P}$ angestellt haben. 
Wenn wir diesen 'leil unserer Untersuchungen allein betrachten würden, ohne dass uns die Daten der vorigen 110 Tage zur Verfügung ständen, so könnten wir, wie die Tabellen zeigen, sagen, dass das Calc. lact. wie auch das $\mathrm{P}$ und Cale. phosphat. die Retention der Mineralstoffe nicht günstig beinflusst hat.

Retention in Prozcht der Aufnahme.

\begin{tabular}{|c|c|c|c|c|c|c|c|}
\hline & $\begin{array}{l}\text { Vorperiode } \\
\text { Adrenalin- } \\
\text { pause }\end{array}$ & Calc. lact. & Pause & Phosphor & Pause & $\begin{array}{c}\text { Trikalzium- } \\
\text { phosphat }\end{array}$ & Pallse \\
\hline $\mathrm{N}$ & 13,2 & $-10,4$ Verlust & 15,6 & - 3,46 Verlust & 7,62 & 4,01 & 3,45 \\
\hline $\begin{array}{l}\mathrm{Ca} \\
\mathrm{Mg}\end{array}$ & $\begin{array}{l}13,1 \\
30,2\end{array}$ & $\begin{array}{c}19,3 \\
-\quad 6,97\end{array}$ & $\begin{array}{l}9,44 \\
3,86 \text { Verlust }\end{array}$ & $\begin{array}{l}3,6 \\
-16\end{array}$ & $\begin{array}{l}-4,06 \text { Verlust } \\
285\end{array}$ & $\begin{aligned} & 36,1 \\
- & 227 \text { Verlust }\end{aligned}$ & $\begin{array}{l}-19,6 \text { Verlust } \\
-\quad 694\end{array}$ \\
\hline $\mathrm{p}$ & 59,9 & 25,1 & 23,4 & 10,5 & 14,0 & 23,5 & 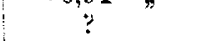 \\
\hline
\end{tabular}

Sogar in der Versuchsperiode des $\mathrm{P}$, aber noch eher in der nach dem Calc. phosphor. folgenden letzten Phase ist es der Ca-Verlust, der sehr stark auffällt. Das, was wir ausserdem für wichtig halten zu bemerken, das ist, dass weder das Ca-Salz, noch das P oder Calc. phosphor. im stande sind, die Retention der untersuchten Mineralstoffe günstig zu beeinflussen.

\begin{tabular}{|c|c|c|c|c|c|}
\hline \multirow{2}{*}{$\begin{array}{c}\text { Dauer des Versuches } \\
\text { 39 Tage } \\
\text { 2. 3.-9. 4. } 1914\end{array}$} & \multirow[b]{2}{*}{ Einf $u h r$} & \multicolumn{3}{|c|}{ Ausscheidung } & \multirow[b]{2}{*}{ Resultat } \\
\hline & & Urin & Fäzes & $\begin{array}{c}\mathrm{Zu}- \\
\text { sammen }\end{array}$ & \\
\hline $\begin{array}{c}\mathrm{N} \\
\mathrm{Ca} \\
\mathrm{Mg}\end{array}$ & $\begin{array}{r}486,78 \\
77,03 \\
9,05 \\
75,22\end{array}$ & \begin{tabular}{|c|}
441,88 \\
3,02 \\
1,80 \\
$33,77(?)$
\end{tabular} & $\begin{array}{r}30,04 \\
63,74 \\
7,60 \\
25,38\end{array}$ & $\begin{array}{r}47], 92 \\
66,76 \\
9,40 \\
59,15\end{array}$ & $\begin{array}{l}+14,86 \\
+10,27 \\
-0,35 \\
+16,07(?)\end{array}$ \\
\hline
\end{tabular}

Also die Zusammenfassung des zweiten (39 tägigen) Teiles unserer Versuchsserie, weist schon nicht jenes günstige Verhältnis auf, wolches der erste Teil zeigte.

Die Bilanz des Mg endet mit Verlust. Auch die des Ca würde mit Verlust enden, wenn wir das Quantum des extra zugeführten $\mathrm{Ca}$ von der Gesamtretention abziehen würden. Allerdings, wenn wir unsere sämtlichen auf 149 Tage sich beziehenden Daten summieren würden, so würde im Endergebnis auch die Bilanz des $\mathrm{Mg}$ mit bedeutendem Gewinne enden. In diosem letzten Teile unseres Versuches können wir schen, dass die Steigerung der Ausscheidung nicht nur mit den Fäzes, sondern bis zu einem bestimmten Grade und im Verhältnis auch mit dem Urin geschehen ist. Von unseren Daten erwähnen wir auch noch das, was wir bei der Anfangsperiode schon hervorgehoben haben, dass neben der hochgradigen Eiweissretention, die Retention des P-Elementes nicht entsprechend gross ist, im Hinblick auch auf die noch ziemlich hohe Ca-Retention (die Retention beträgt $24,4 \mathrm{pCt}$. der Einfuhr beim P, bei $\mathrm{Ca} 67,4$ pCt.) und wir betonten, dass es ebenso wichtig ist das Verhalten des P-Elementes zu kennen, wie das der übrigen knochenaufbauenden Stoffe. Während unserer Untersuchungen, welche 149 Tage umfassen, haben wir in keiner Periode einen P-Verlust gesehen, obzwar 
wir unter unseren Daten auch solche Serien finden konnten, in denen neben Eiweissverlust $\mathrm{Ca}$-, ja sogar $\mathrm{Mg}$-Verlust zu beobachten war. Unsere letzte Periode könnte man vielleicht als solche betrachten, wo die Bilanz des $\mathbf{P}$ mit Verlust geendet hat; aber wir können auch diese Annahme nicht beweisen, weil wir gerade in dieser Periode den P-Gehalt des Urins nicht bestimmt haben.

Wir halten es nicht für ausgeschlossen, dass sich die Besserung des Mineralstoffwechsels bei Osteomalazie mit der Besserung der Retention des $\mathrm{Mg}$, dann des $\mathrm{Ca}$ und nur nachher mit der des $\mathrm{P}$ ordnet, während. bei der Verschlechterung des Mineralstoffwechsels in erster Reihe in der Verwendung des Mg-Elementes, dann in der des Ca eine Störung besteht, und dass die Retention des $\mathrm{P}$ die hartnäckigste ist. Dies ist indessen eine Annahme, die wir aus unseren Daten herauszulesen meinen. Jede Verallgemeinerung steht uns fern.

Wir müssen noch erwähnen, dass wir in den ersten drei Monaten die Retention der $\mathrm{Ca}$-, Mg- und P-Elemente nicht beeinflussen konnten, ebenso stand es nicht in unserer Macht, in den letzten drei Serien den ungünstigen Gang des Mineralstoffwechsels während der Versuchszeit zu modifizieren, nur gerade provisorisch; obzwar in diesen Serien unser Eingreifen schon viel energischer war (täglich $4 \mathrm{~g}$ Calc. lact., dann täglich 5 g Calc. phosphat.). Diese Daten beweisen es zur Genüge, dass, wie bei Rachitis, ebenso auch bei Osteomalazie die Störungen des Mineralstoffwechsels die charakteristischen Symptome der Krankheit bilden.

Darüber, ob diese Mineralstoffwechselstörung als pathogenetische Ursache oder Folgerung betrachtet werden soll, würden wir uns - auf Grund unserer geringen Erfahrungen - nicht zu äussern wagen.

\section{Literaturverzeichnis.}

Abderhalden, Lehrbuch der physiologischen Chewie. 1915. 3. Auf. 2. Teil.Albu u. Neuberg, Physiologie und Pathologie des Mineralstoffwechsels. Berlin 1906. - Bauer, Die konstitutionelle Disposition zu inneren Krankheiten. Berlin 1917. Basch, Thymus in Lehrbuch der Organotherapie. Wagner v. Jauregg. Leipzig 1914. - R. Bieling, Der Einfluss von Extrakten endokriner Drüsen auf den Mineralstoffwechsel und das Blutbild rachitischer Säuglinge. Biochem. Zeitschr, 1914. Bd. 63. S. $95 \mathrm{ff}$. - Biedl, Innere Sekretion, 2. Aufl. (Literatur.) Wien u. Berlin 1913. Bossi, Die Nebennieren and Osteomalazie. Arch.f. Gyn. 1907. Bd. 83, (s. Biedl.)Borchardt, L., Hypophyse. Lehrbuch der Organotherapie. Wagnerv. Jauregg. 1914. - Bayer, G., Nebenniere and ehromaffines System. Lehrb. d. Organother. Wagner v. Jauregg. 1914. - Bab, H., Die Behandlung der Osteomalazie mit Hypophysenextrakt. Münchener med. Wochenschr. 1911. Nr. 34. - E. v.Cyon, Die Gefässdrüsen. Berlin 1910. - Erdbeim, Ueber Epithelkörperbefunde bei Osteomalazie. Sitzungsbericht der k. Akad. zu Wion. 1907. Nr. 116. (Bjedl.) - Elfer, A., Ueber die Wirkung des Extraktes aus dem Infundibularteil der Glandula pituitaria unter pathologischen Verbältnissen. Deutsches Arch. f. klin. Med. 1913. Bd. 110. - Fehling, Ueber Wesen and Behandlung der puerperalen Osteomalazie. Arch. f. Gyn. Bd. 39. S. 183 ff. (Morawitz, Pathologio des Wasser- und Mineralstoffwechsels. S. 320.) Funk, C., Die Vitamine. Wiesbaden 1914. - Falta, Die Erkrankungen der Blutdrüsen. Berlin 1913. - Frangenheim, P., Die Krankheiten des Knochensystems im Kindesalter. Stuttgart 1913. - Gassmann, Chemische Untersuchungen von go- 
128 A. Elfer u. J. Kappel, Daten z. Wirkung d. Extrakte einiger innerer Drüsen usw.

sunden und rachitischen Knochen. Zeitschr. f. physiol. Chem. Bd. 70. - H. Matti, Physiologie und Patbologie der Thymusdrüse. Ergebn. d. inneren Med. u. Kinderheilkunde. 1913. Bd. 10. - L. Mohr, Erkrankungen der Knochen und Gelenke. Handb. d. Pathol. d. Stoffwechsels. 1907. 2. Bd. -- Morawitz, P., Pathologie des Wasser- und Mineralstoffwechsels. Handb. d. Biochem. 1910. Bd. 4, 2. A. - Neumann, S., A Calc. Mg. és phosphorsav kiválasztásának viszonyairól Osteomalaciarial. Magy. Orv. Aroh, 1894. Bd.3. S. 211. - Neumann, S. és Vas B., A Cale. és Ma. kiválasztásáról ép és kóros viszoyyok kózótt. Magy. Orv. Arch. 1894. Bd. 3. S. 305 ff. - Neumann, S., Weitere Untersuchungen über den Stoffwechsel usw. Arch. f. Gyn. Bd. 51. - Loew, O., Die Lehre rom Kalkfaktor. Berlin 1914. -- Derselbe, Zur chemischen Physiologie des Kalks bei Menschen und Tieren. München 1916. --Lehnerdt, Fr., Warum bleibt das racbitische Knochengewebe unverkalkt. Ergebn. d. inn. Med, u. Kinderbeilk. 1910. Bd. 6. - Orgler, Der Kalkstofiwechsel des gesunden und rachitischen Kindes. Ebenda. 1912. Bd. 8. - Pommer, G., Untersuchungen über Osteomalazie und Racbitis. Leipzig 1885. - K. v.Recklinghausen, Untersuchungen über Rachitis und Osteomalazie. Jena 1910. - Schmorl, Die pathologische Anatomie der rachitischen Knochenerkrankungen mit besonderer Berücksichtigung ihrer Histologie und Pathogenese. Ergebn. d. inn. Med. u. Kinderheilk. 1909. Bd. 4. - E. Schloss, 80 Stoffwechselversuche über die therapeutische Beeinflussung des rachitischen Stoffwechsols. Berlin 1916. - Schloss, E., Dio Pathogenese und Aetiologie der Rachitis sowie die Grundlagen ihrer Therapie. Ergebn. d. inner. Med, v. Kinderheilk. 1917. Bd. 15. - Scipiades, E., Az osteo malaciáról. Orvosi Hetilap. 1917. 37 és 38 sz. - Trousseau, A., Medizinische Klinik des Hôtel Dieu in Paris. Nach der 2. Aufl. deutsch bearbeitet von Dr. L. Culmann, fortg. von P. Niemeyer. Würzburg 1868. Bd.3. S. 387. - Wieland, Die Frage der angeborenen und der hereditären Rachitis. Ergebn. d. inn. Mediz. u. Kinderheilk. 1910. Bd. 6. - Derselbe, Rachitis tarda. Ebenda. 1914. Bd.13. - Derselbe, Spezielle Pathologie dos Bewegungsapparates im Kindesalter. (Brünnig-Schwalbe, Handb. d. allg. P. u. P. A. des Kindesalters. 1913. 2. Bd. 1. Abt.) - Woblauer, F., Atlas und Grundriss der Rachitis. München 1911. - G. v. Wendt, Mineralstoffwechsel. Handb. d. Biochem. 1911. Bd. 4. H. 1. 